\title{
Palladium-Catalyzed Oxidative Cross-Coupling of Conjugated Enynones with Allylarenes: Synthesis of Furyl-Substituted
}

\author{
1,3-Dienes \\ Yifan Ping, ${ }^{a}$ Sudong Zhang, ${ }^{a}$ Taiwei Chang, ${ }^{a}$ and Jianbo Wang* ${ }^{a, b}$ \\ ${ }^{a}$ Beijing National Laboratory of Molecular Sciences (BNLMS) and Key Laboratory of \\ Bioorganic Chemistry and Molecular Engineering of Ministry of Education, College of \\ Chemistry, Peking University, Beijing 100871, China \\ ${ }^{b}$ The State Key Laboratory of Organometallic Chemistry, Shanghai Institute of Organic \\ Chemistry, Chinese Academy of Sciences, Shanghai 200032, China \\ Email:wangjb@pku.edu.cn
}

1. Mechanistic Studies .

2. References. S4

3. ${ }^{1} \mathrm{H}$ and ${ }^{13} \mathrm{C}$ NMR Spectra S5 


\section{Mechanistic Studies}

\section{KIE Experiment (Intramolecular Competitive Experiment)}

[D $\mathbf{1}$-2d was synthesized according to the literature procedure. ${ }^{1}$<smiles>CC(=O)C(=CC#CBr)C(C)=O</smiles>

1a<smiles>[2H]C(C)(C=C)c1ccc(-c2ccccc2)cc1</smiles>

$\left[D_{1}\right]-2 d$

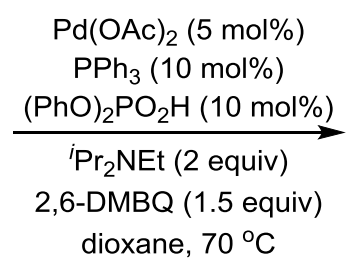

dioxane, $70^{\circ} \mathrm{C}$<smiles>CC(=O)c1cc(/C(=C\C=C\c2ccc(-c3ccccc3)cc2)[Hg]Oc2ccccc2)oc1C</smiles>

$\left[D_{1}\right]-3 d: 3 d=2.7$

$\mathrm{Pd}(\mathrm{OAc})_{2}(2.2 \mathrm{mg}, 0.01 \mathrm{mmol}, 5 \mathrm{~mol} \%), \mathrm{PPh}_{3}(5.3 \mathrm{mg}, 0.02 \mathrm{mmol}, 10 \mathrm{~mol} \%)$, $(\mathrm{PhO})_{2} \mathrm{PO}_{2} \mathrm{H}(5.0 \mathrm{mg}, 0.02 \mathrm{mmol}, 10 \mathrm{~mol} \%)$ and 2,6-dimethyl-1,4-benzoquinone (40.8 mg, $0.3 \mathrm{mmol}, 1.5$ equiv) were added to a flame-dried $10 \mathrm{~mL}$ Schlenk reaction tube. The reaction tube was degassed three times with nitrogen, then dry dioxane $(2 \mathrm{~mL}, 0.1 \mathrm{M})$ was added using

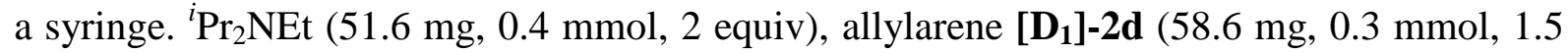
equiv) and conjugated enynone $1 \mathrm{a}(38.4 \mathrm{mg}, 0.2 \mathrm{mmol})$ were added by syringe successively. The reaction tube was stirred at $70{ }^{\circ} \mathrm{C}$ for $16 \mathrm{~h}$, then cooled to room temperature. The mixture was filtered through a short plug of silica gel and washed with $\mathrm{Et}_{2} \mathrm{O}$ as the eluent. Solvent was then removed in vacuo to leave a crude mixture and the products $\left[\mathbf{D}_{\mathbf{1}}\right]-\mathbf{3 d}$ and $\mathbf{3 d}\left(\left[\mathbf{D}_{\mathbf{1}}\right]-\mathbf{3 d}+\right.$ 3d, $56.4 \mathrm{mg}, 74 \%$ ) were isolated by silica gel column chromatography. The ratio of the products $\left(\left[\mathbf{D}_{\mathbf{1}}\right]-\mathbf{3 d} \mathbf{3} \mathbf{3 d}=2.7\right)$ was determined by ${ }^{1} \mathrm{H}-\mathrm{NMR}$ analysis. 


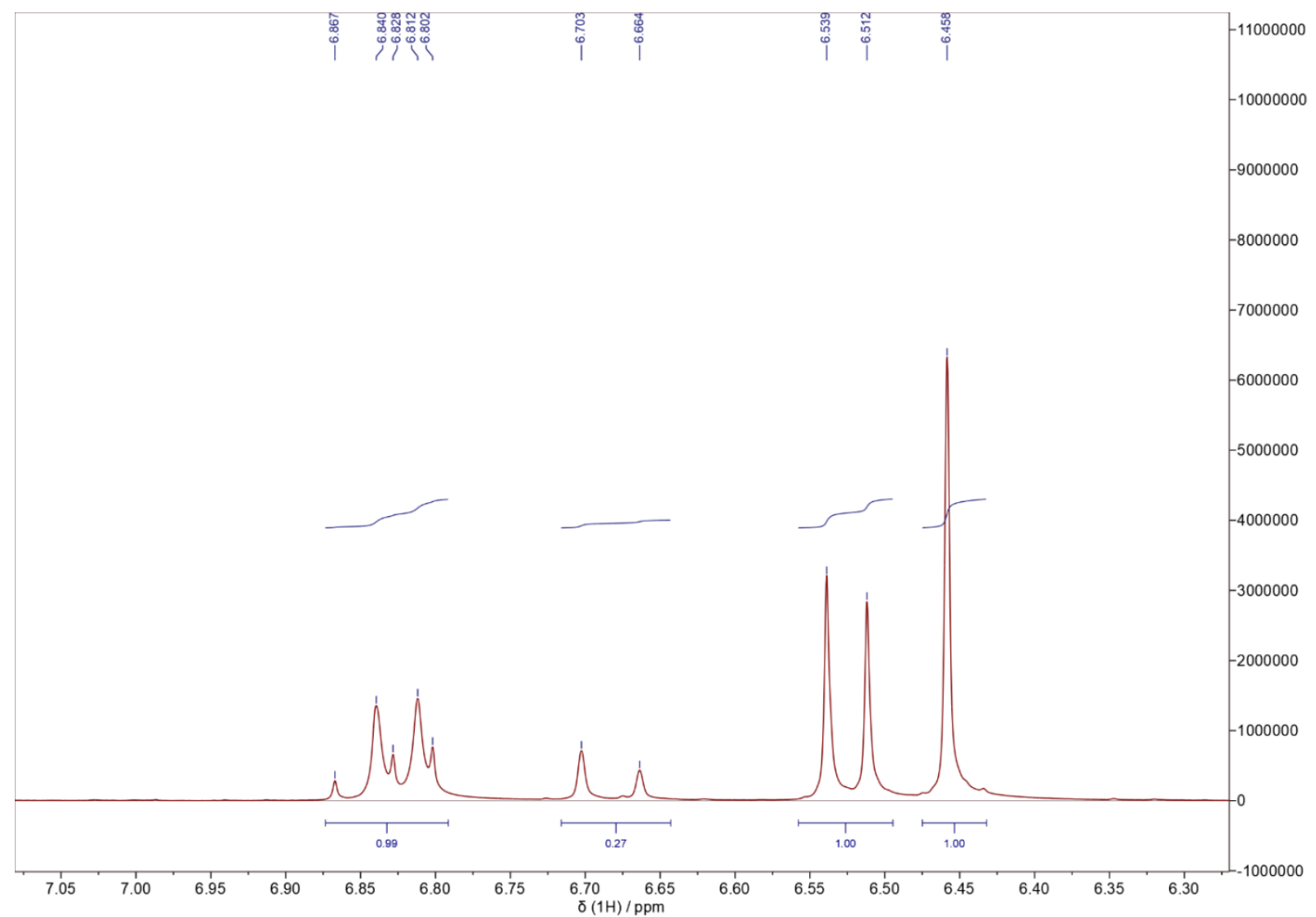

\section{KIE Experiment (Parallel Experiment)}

[D $\left.\mathbf{D}_{2}\right]-2 \mathbf{d}$ was synthesized according to the literature procedure. ${ }^{1}$<smiles>CC(=O)C(=CC#CBr)C(C)=O</smiles>

$1 \mathrm{a}$<smiles>C=CCc1ccc(-c2ccccc2)cc1</smiles>

2d

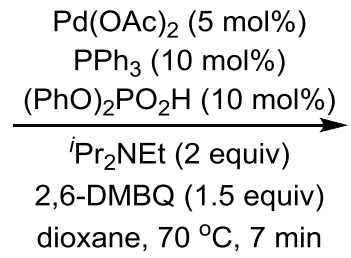

dioxane, $70^{\circ} \mathrm{C}, 7 \mathrm{~min}$

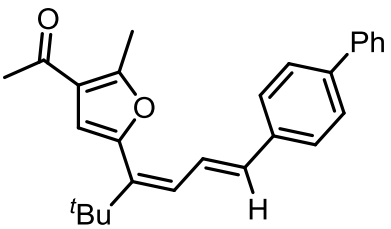

$3 d(11.2 \%$ yield $)$

$\mathrm{Pd}(\mathrm{OAc})_{2}(2.2 \mathrm{mg}, 0.01 \mathrm{mmol}, 5 \mathrm{~mol} \%), \mathrm{PPh}_{3}(5.3 \mathrm{mg}, 0.02 \mathrm{mmol}, 10 \mathrm{~mol} \%)$, $(\mathrm{PhO})_{2} \mathrm{PO}_{2} \mathrm{H}(5.0 \mathrm{mg}, 0.02 \mathrm{mmol}, 10 \mathrm{~mol} \%)$ and 2,6-dimethyl-1,4-benzoquinone (40.8 mg, $0.3 \mathrm{mmol}, 1.5$ equiv) were added to a flame-dried $10 \mathrm{~mL}$ Schlenk reaction tube. The reaction tube was degassed three times with nitrogen, then dry dioxane $(2 \mathrm{~mL}, 0.1 \mathrm{M})$ was added using a syringe. ${ }^{i} \operatorname{Pr}_{2} \mathrm{NEt}$ (51.6 mg, $0.4 \mathrm{mmol}, 2$ equiv), allylarene $\mathbf{2 d}$ (58.3 mg, $0.3 \mathrm{mmol}, 1.5$ equiv) and conjugated enynone 1a $(38.4 \mathrm{mg}, 0.2 \mathrm{mmol})$ were added by syringe successively. The reaction tube was stirred at $70{ }^{\circ} \mathrm{C}$ for $7 \mathrm{~min}$, then cooled to room temperature. The mixture was filtered through a short plug of silica gel and washed with $\mathrm{Et}_{2} \mathrm{O}$ as the eluent. The yield of 3d was determined by ${ }^{1} \mathrm{H}-\mathrm{NMR}$ using dibromomethane $(17.4 \mathrm{mg}, 0.1 \mathrm{mmol})$ as the internal standard (NMR yield: $11.2 \%)$. 

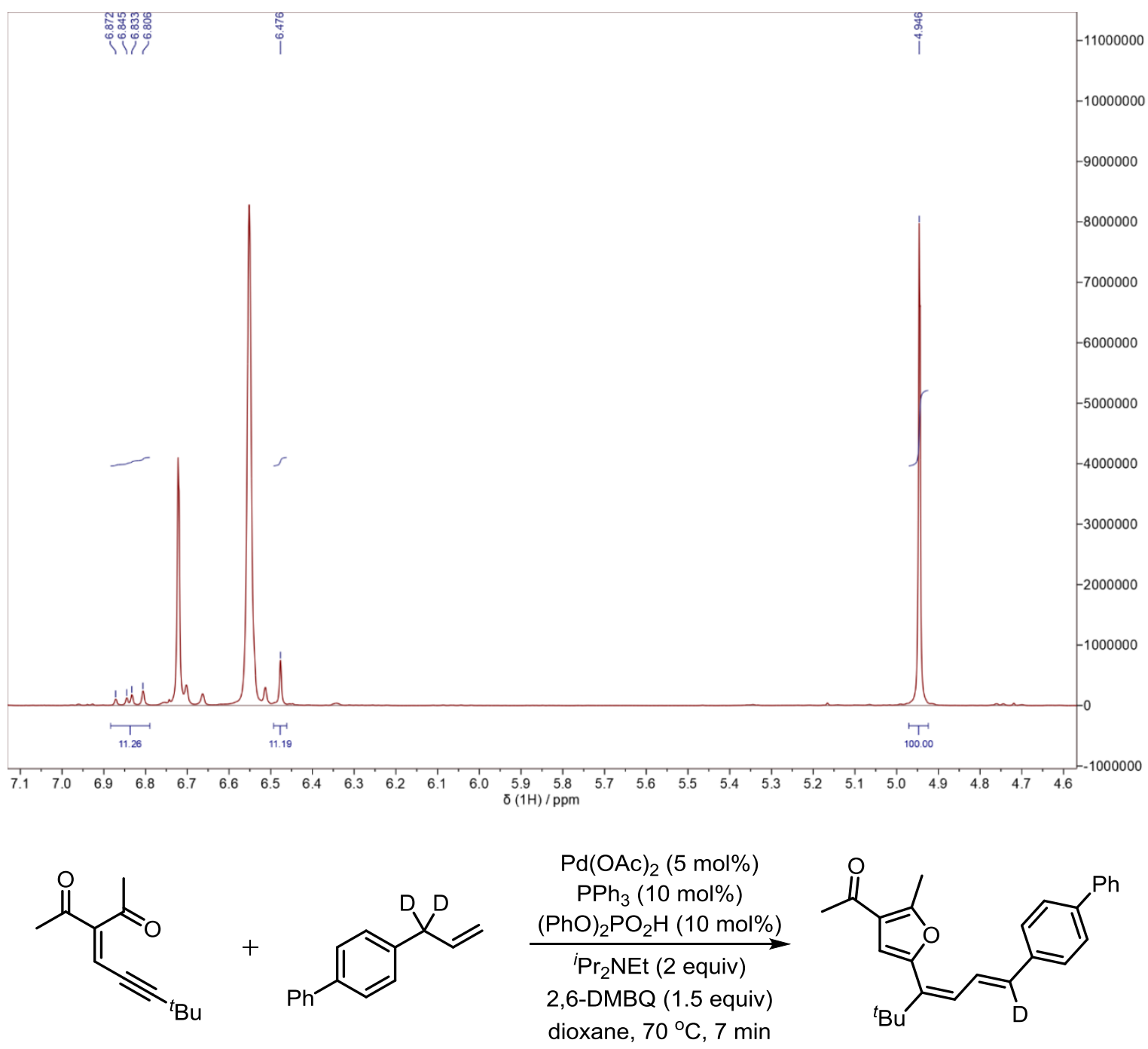

$1 \mathrm{a}$

$\left[D_{2}\right]-2 d$

$\left[D_{1}\right]-3 d(5.9 \%$ yield)

$\mathrm{Pd}(\mathrm{OAc})_{2}(2.2 \mathrm{mg}, 0.01 \mathrm{mmol}, 5 \mathrm{~mol} \%), \mathrm{PPh}_{3}$ (5.3 mg, $\left.0.02 \mathrm{mmol}, 10 \mathrm{~mol} \%\right)$, $(\mathrm{PhO})_{2} \mathrm{PO}_{2} \mathrm{H}(5.0 \mathrm{mg}, 0.02 \mathrm{mmol}, 10 \mathrm{~mol} \%)$ and 2,6-dimethyl-1,4-benzoquinone (40.8 mg, $0.3 \mathrm{mmol}, 1.5$ equiv) were added to a flame-dried $10 \mathrm{~mL}$ Schlenk reaction tube. The reaction tube was degassed three times with nitrogen, then dry dioxane $(2 \mathrm{~mL}, 0.1 \mathrm{M})$ was added using a syringe. ${ }^{i} \operatorname{Pr}_{2} \mathrm{NEt}\left(51.6 \mathrm{mg}, 0.4 \mathrm{mmol}, 2\right.$ equiv), allylarene [ $\left.\mathbf{D}_{\mathbf{2}}\right]-\mathbf{2 d}(58.9 \mathrm{mg}, 0.3 \mathrm{mmol}, 1.5$ equiv) and conjugated enynone 1a $(38.4 \mathrm{mg}, 0.2 \mathrm{mmol})$ were added by syringe successively. The reaction tube was stirred at $70{ }^{\circ} \mathrm{C}$ for $7 \mathrm{~min}$, then cooled to room temperature. The mixture was filtered through a short plug of silica gel and washed with $\mathrm{Et}_{2} \mathrm{O}$ as the eluent. The yield of $\left[\mathbf{D}_{\mathbf{1}}\right] \mathbf{- 3 d}$ was determined by ${ }^{1} \mathrm{H}-\mathrm{NMR}$ using dibromomethane $(17.4 \mathrm{mg}, 0.1 \mathrm{mmol})$ as the internal standard (NMR yield: 5.9\%). 


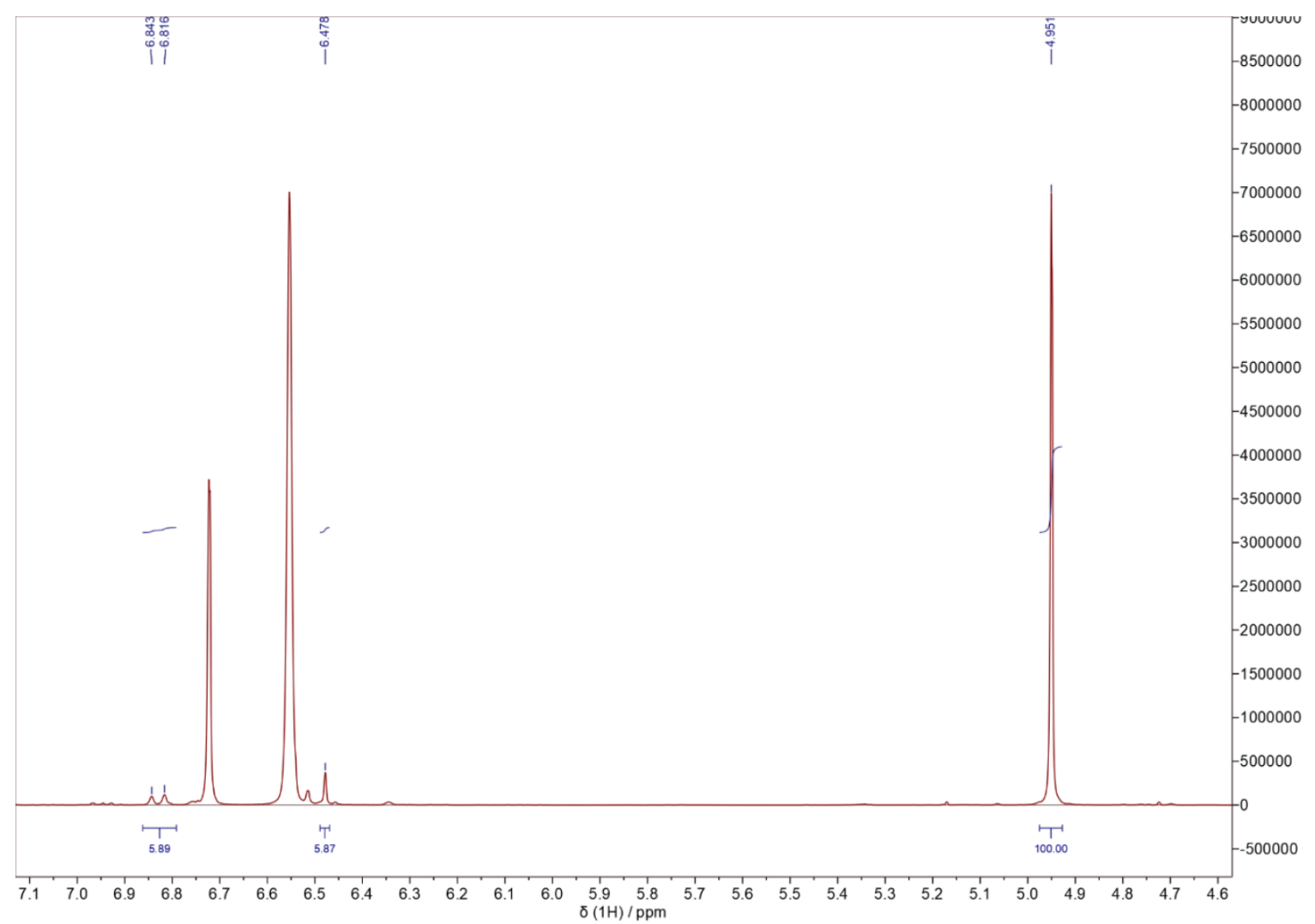

These two reactions were conducted at the same time in the same oil bath. KIE = 11.2/5.9 $=1.9$. Such parallel experiments were also performed for $5 \mathrm{~min}$ and $10 \mathrm{~min}$, similar KIE were observed.

\begin{tabular}{|c|c|c|c|}
\hline Reaction time & Yield $^{a}(\mathbf{3 a})$ & Yield $^{a}$ & KIE \\
\hline 5 min & $4.7 \%$ & $2.6 \%$ & 1.8 \\
\hline 7 min & $11.2 \%$ & $5.9 \%$ & 1.9 \\
\hline $10 \mathrm{~min}$ & $14.8 \%$ & $7.8 \%$ & 1.9 \\
\hline
\end{tabular}

${ }^{a}$ Determined by ${ }^{1} \mathrm{H}-\mathrm{NMR}$ using dibromomethane as the internal standard

\section{References}

(1) Yin, G.; Wu, Y.; Liu, G. Scope and Mechanism of Allylic C-H Amination of Terminal Alkenes by the Palladium/PhI(OPiv $)_{2}$ Catalyst System: Insights into the Effect of Naphthoquinone. J. Am. Chem. Soc. 2010, 132, 11978. 


\section{3. ${ }^{1} \mathrm{H}$ and ${ }^{13} \mathrm{C}$ NMR Spectra}

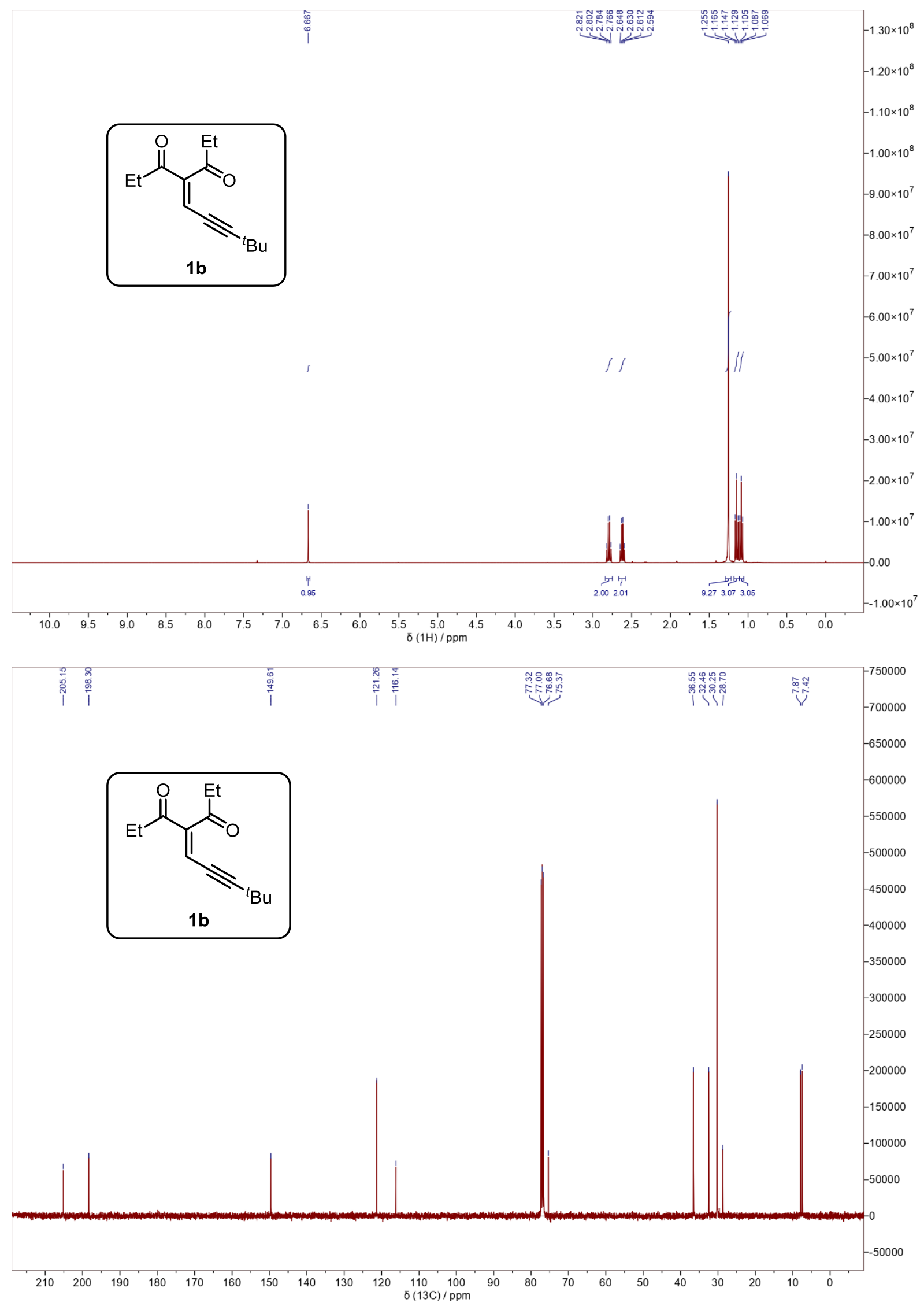




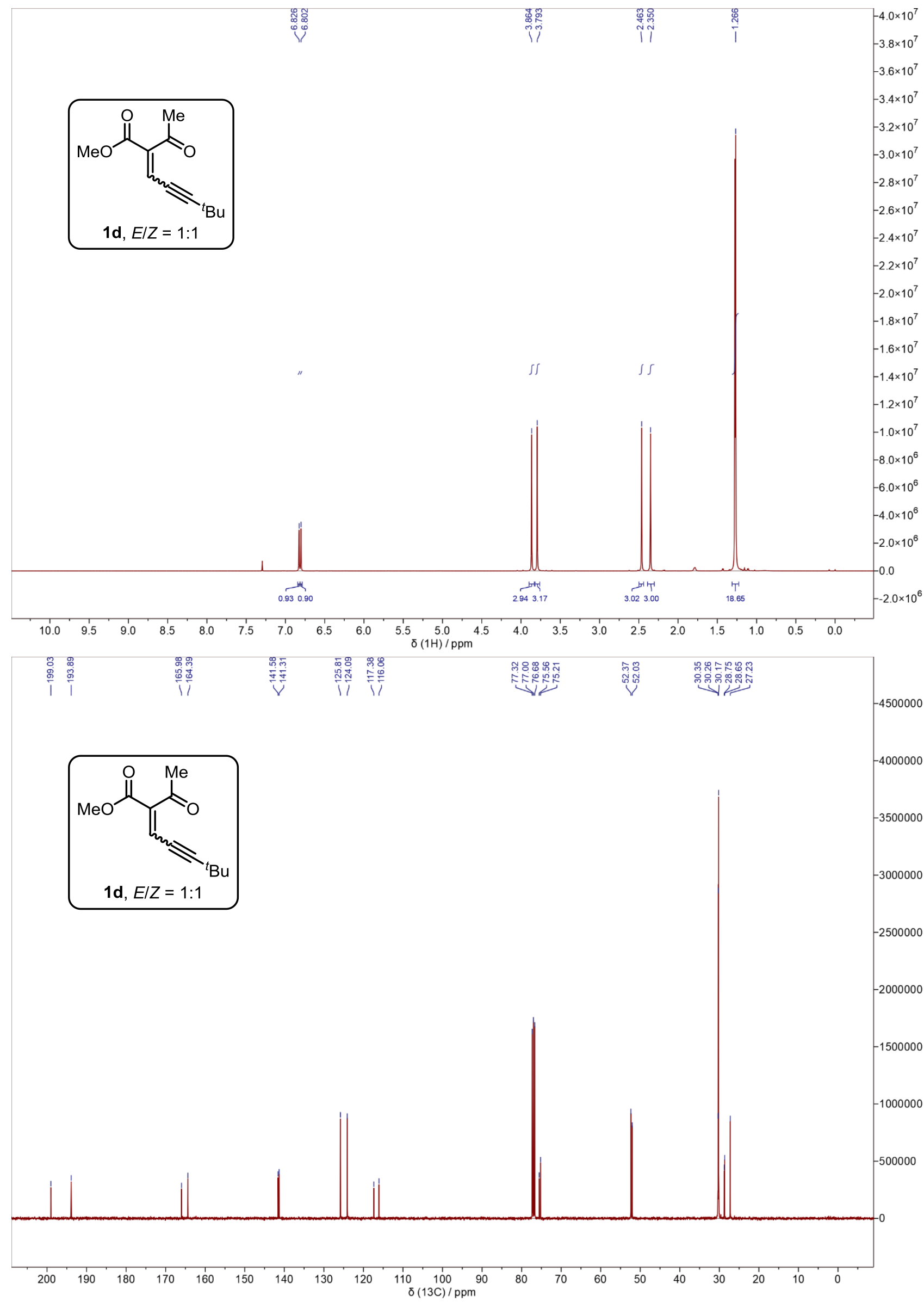




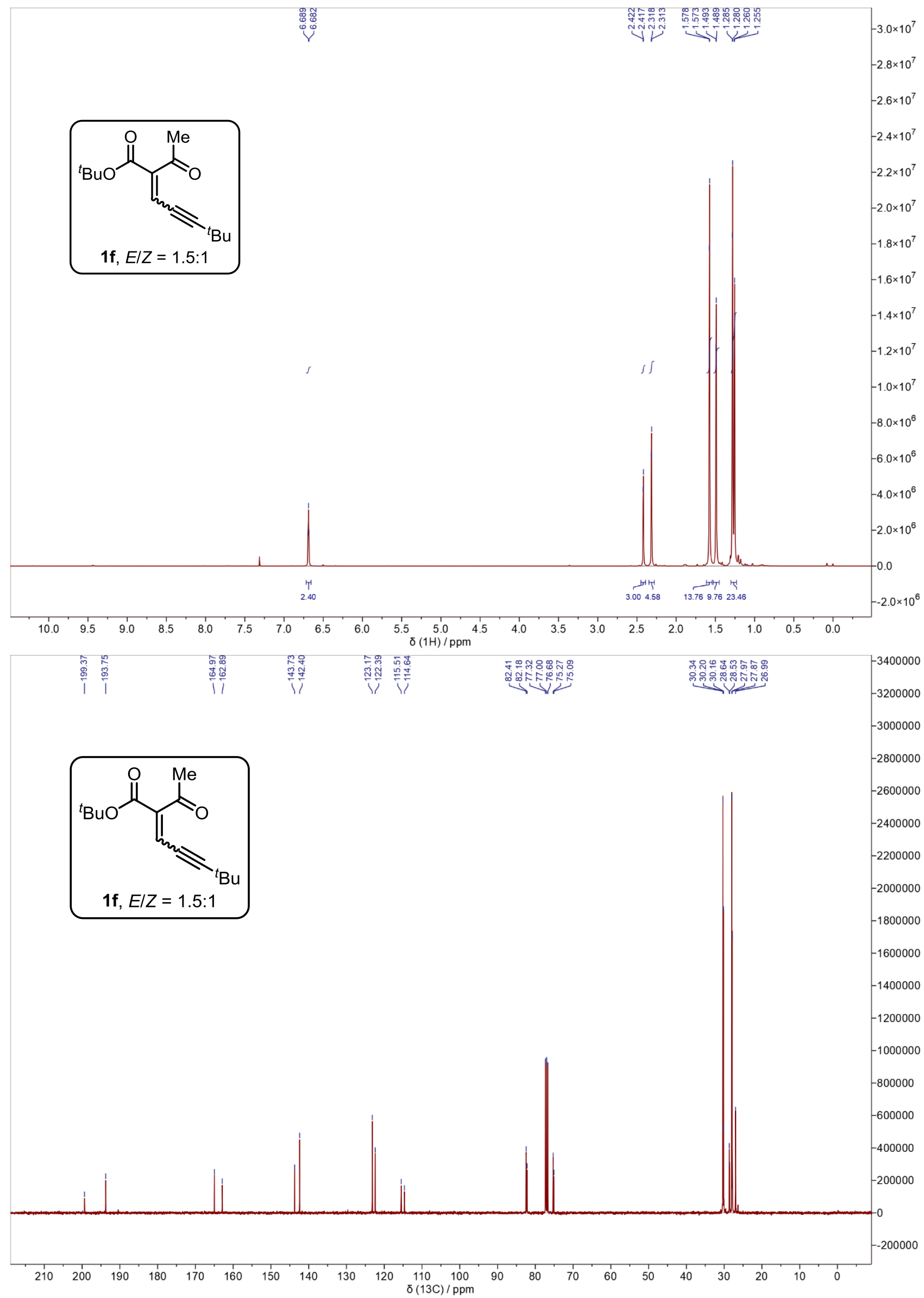




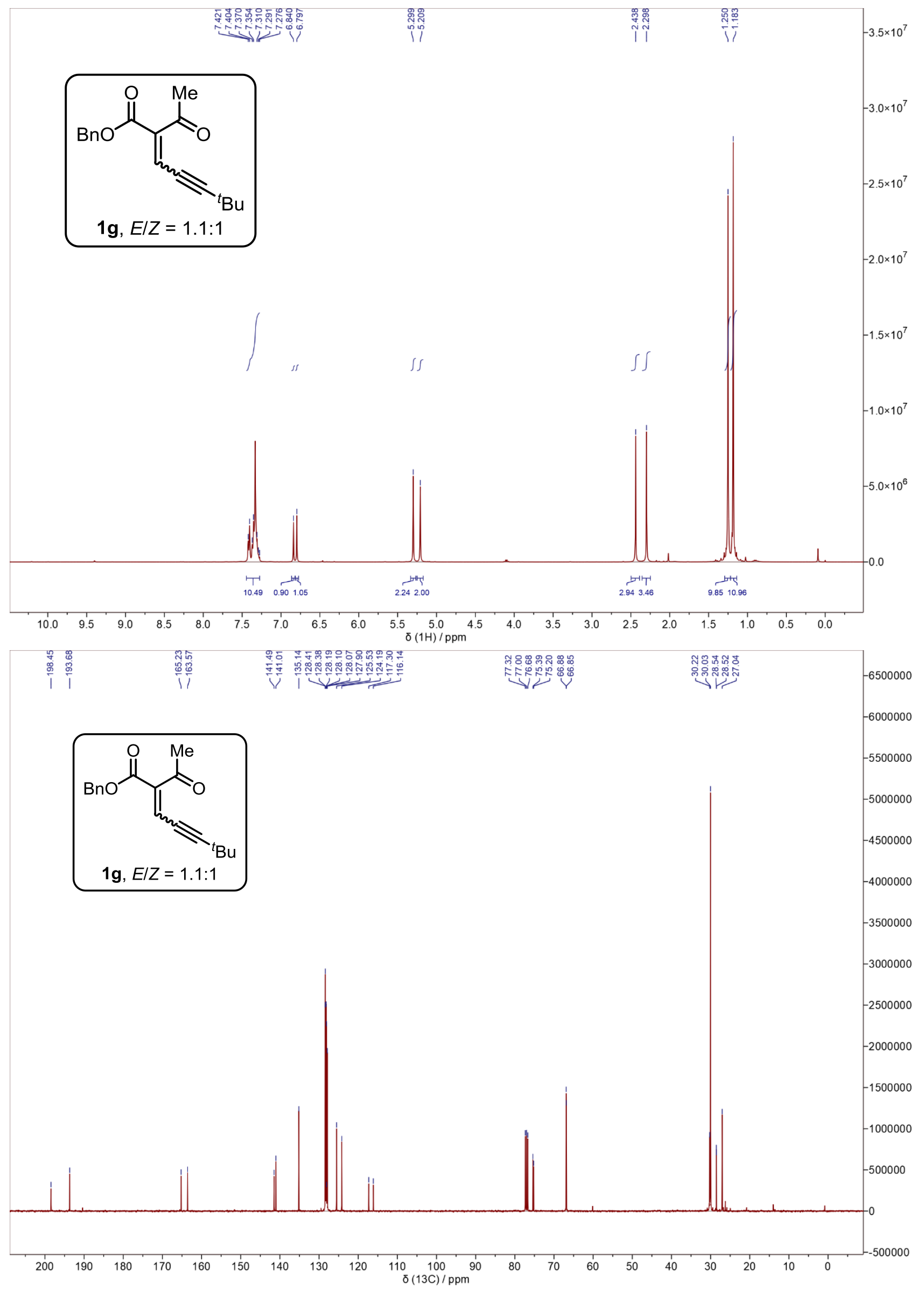




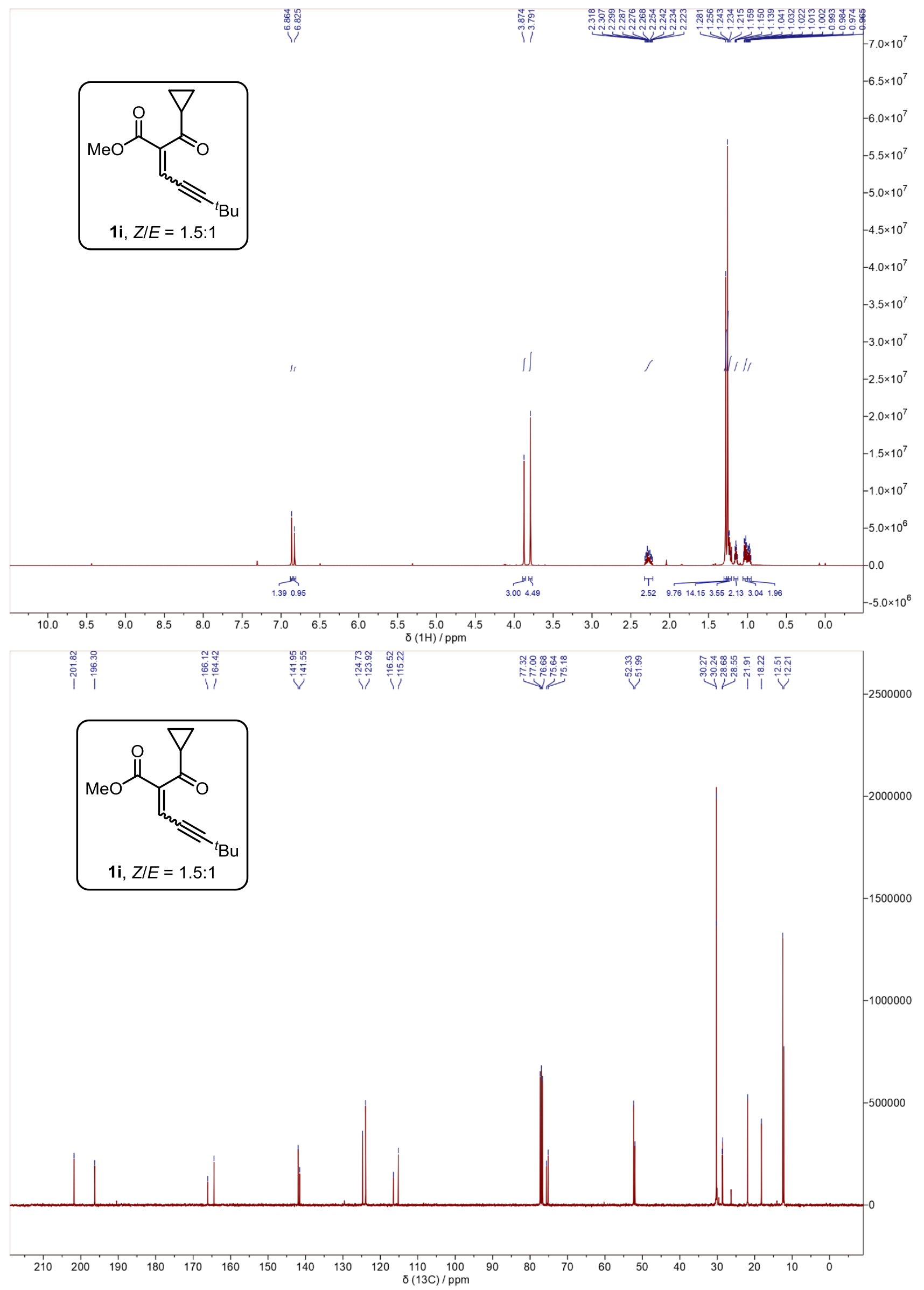




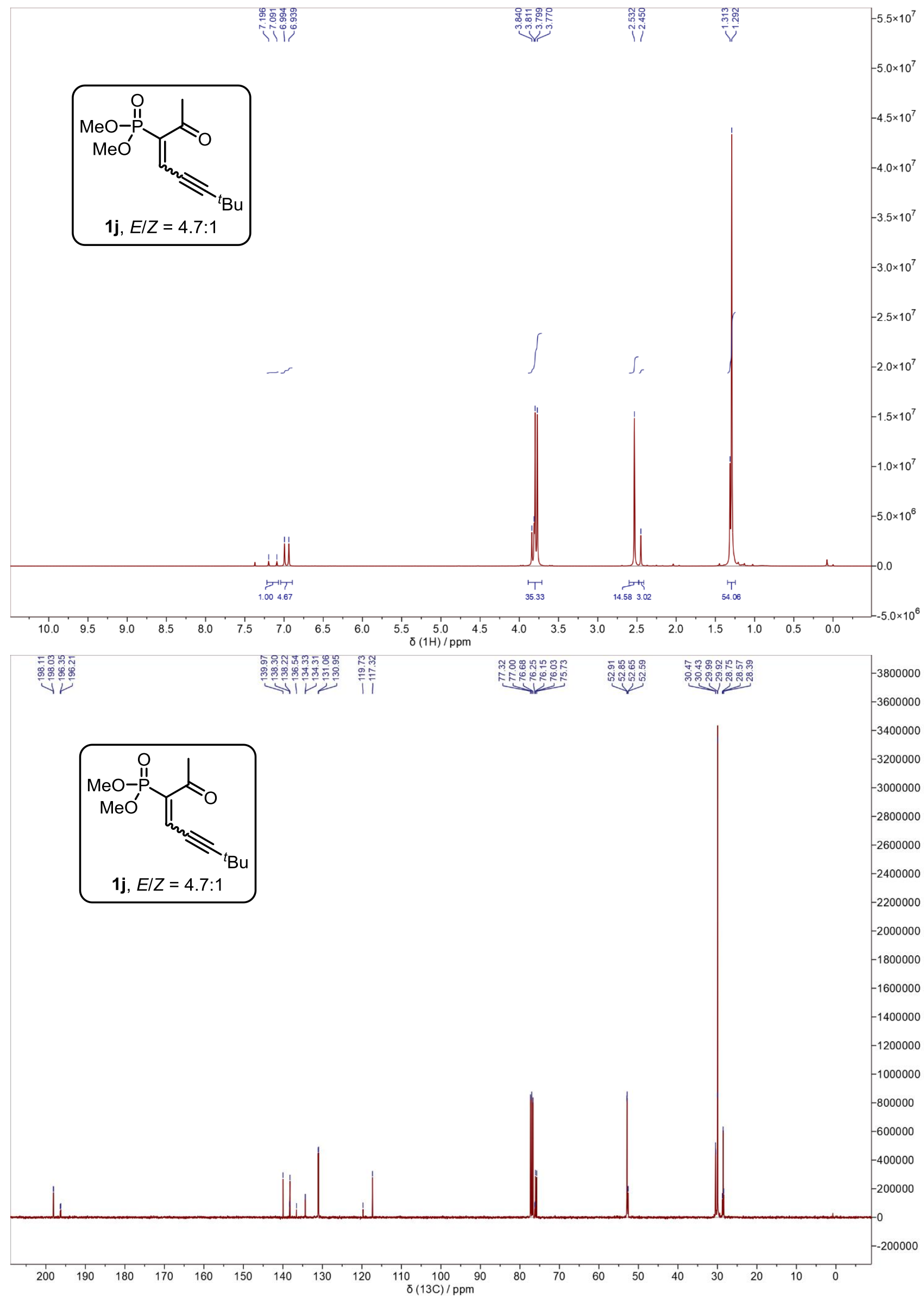




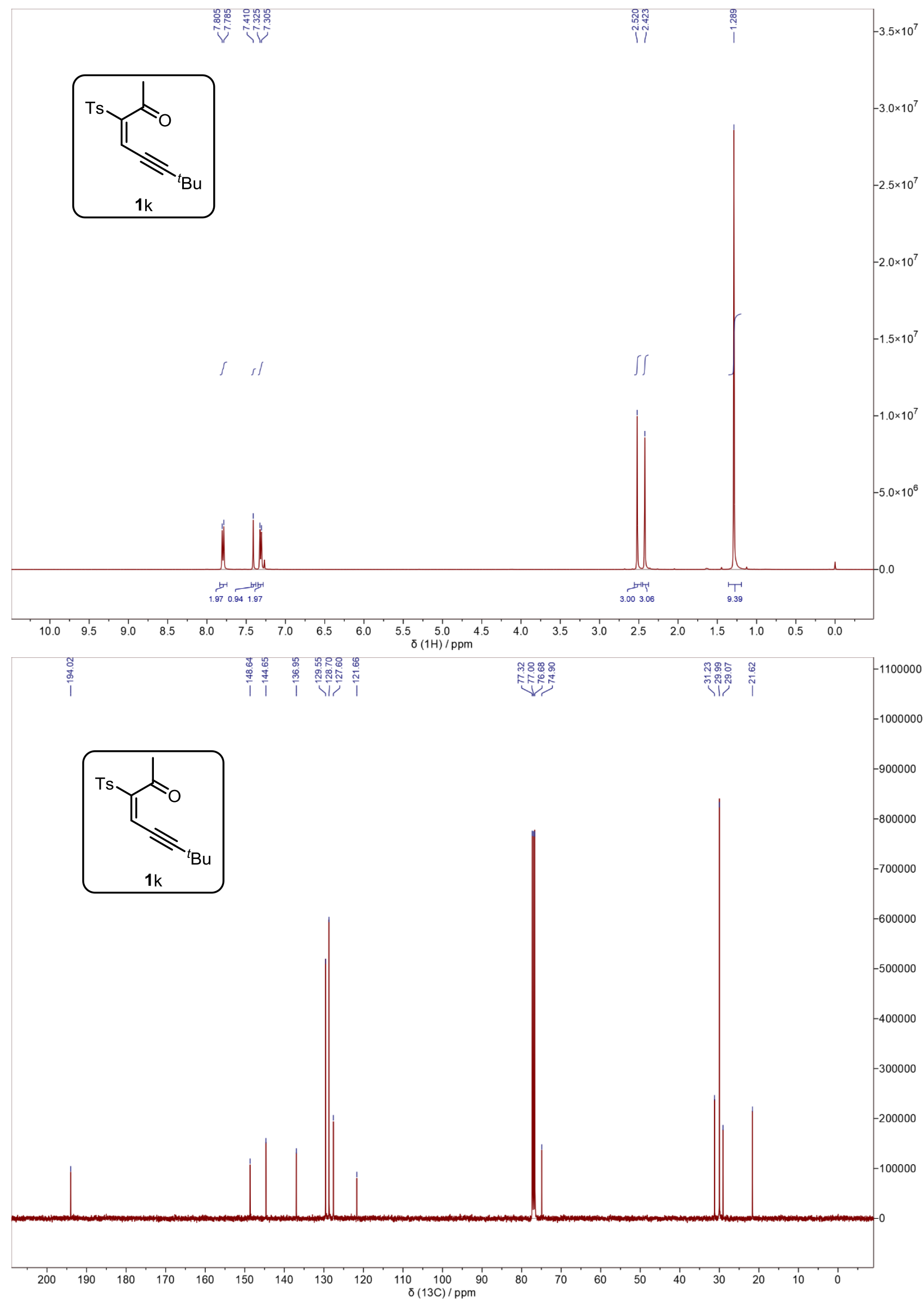




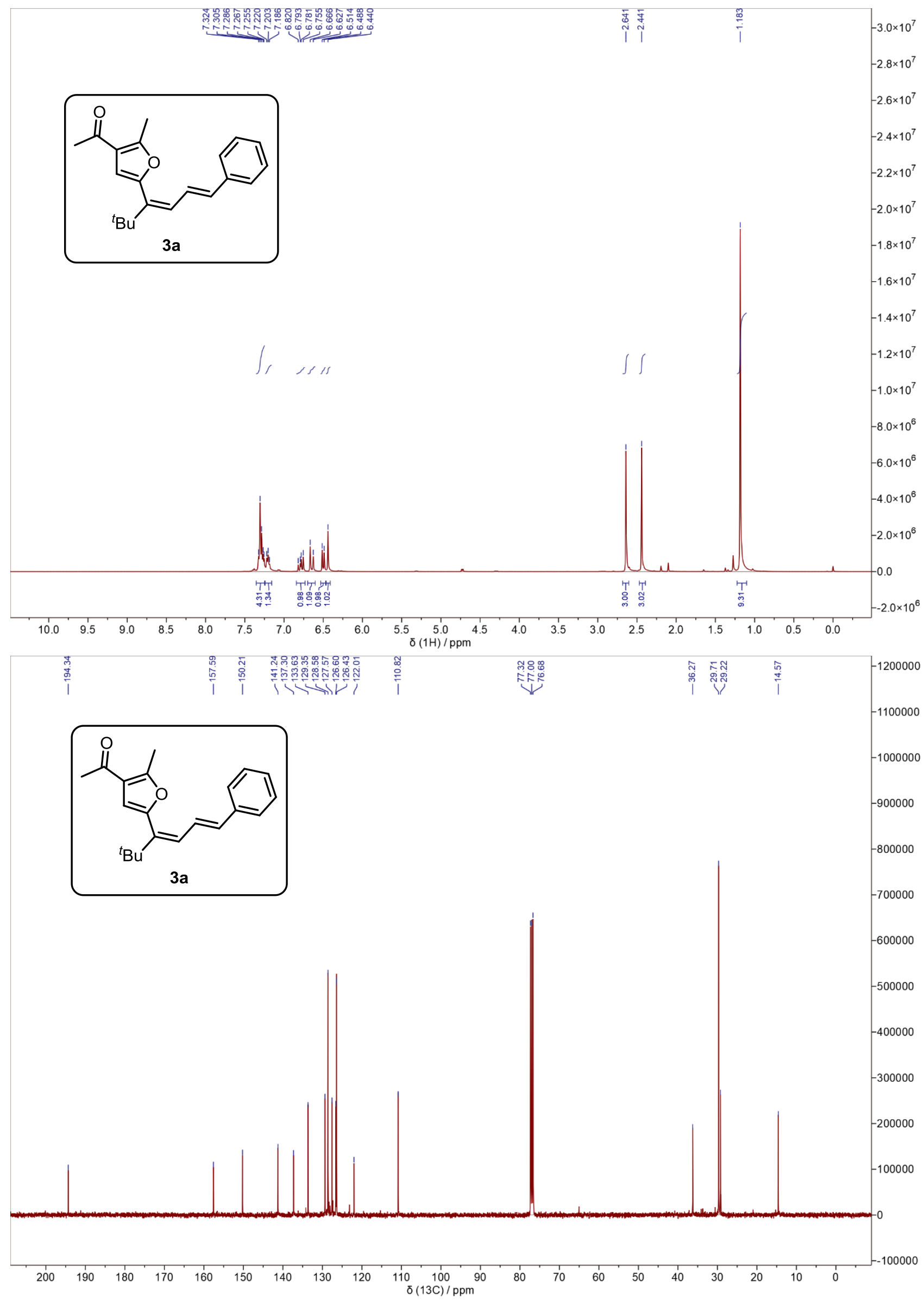




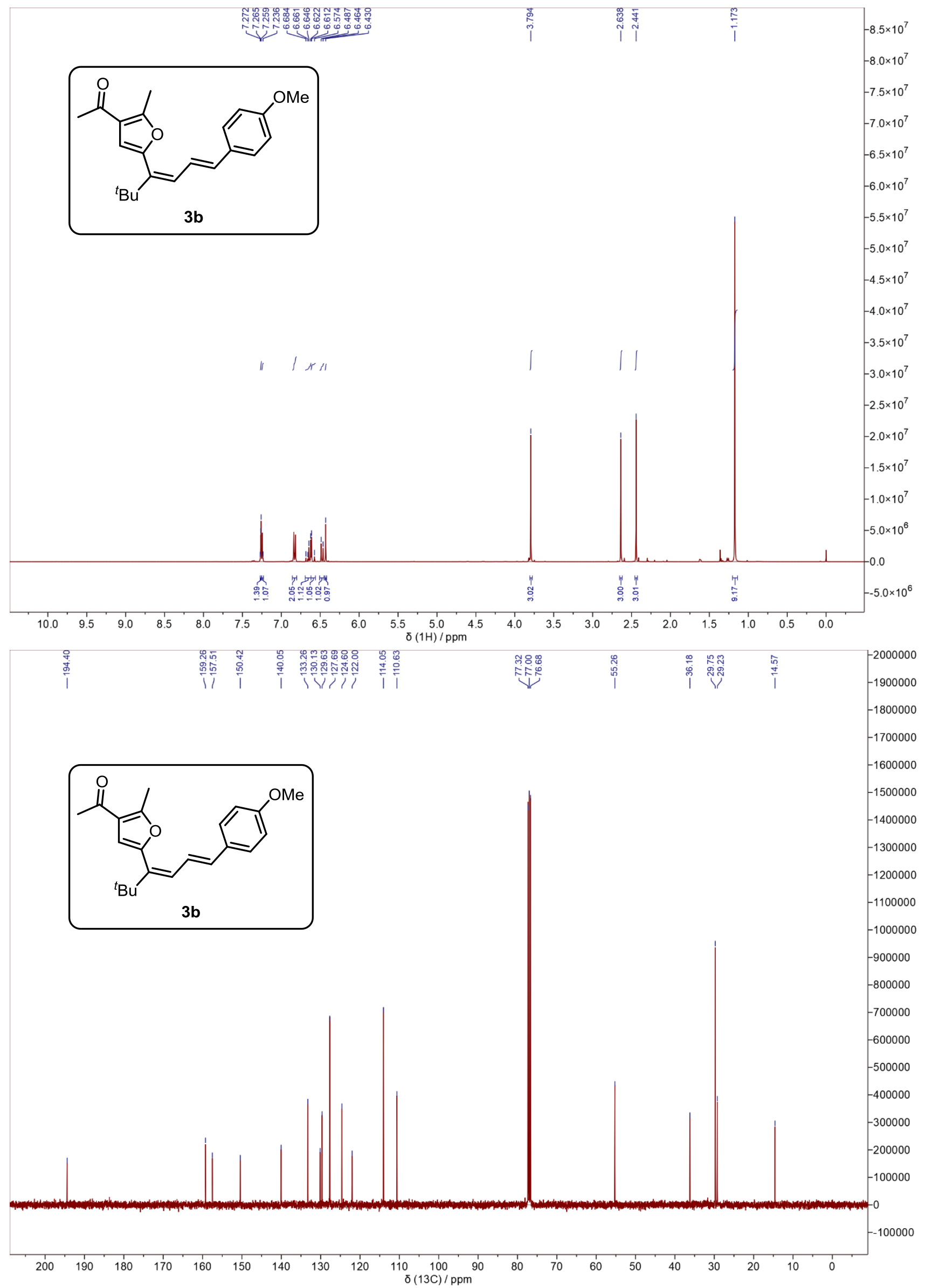

S13 


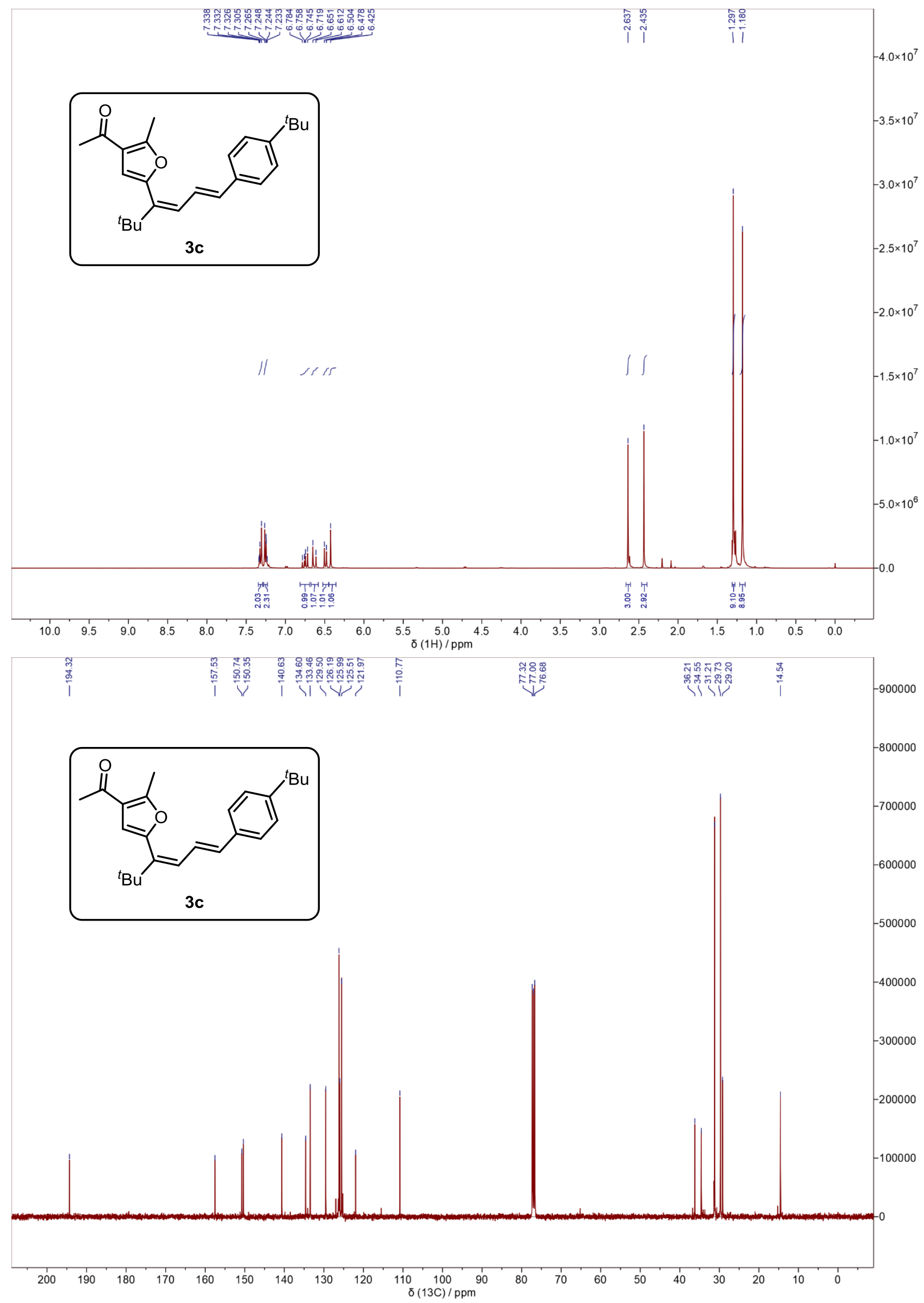

S14 


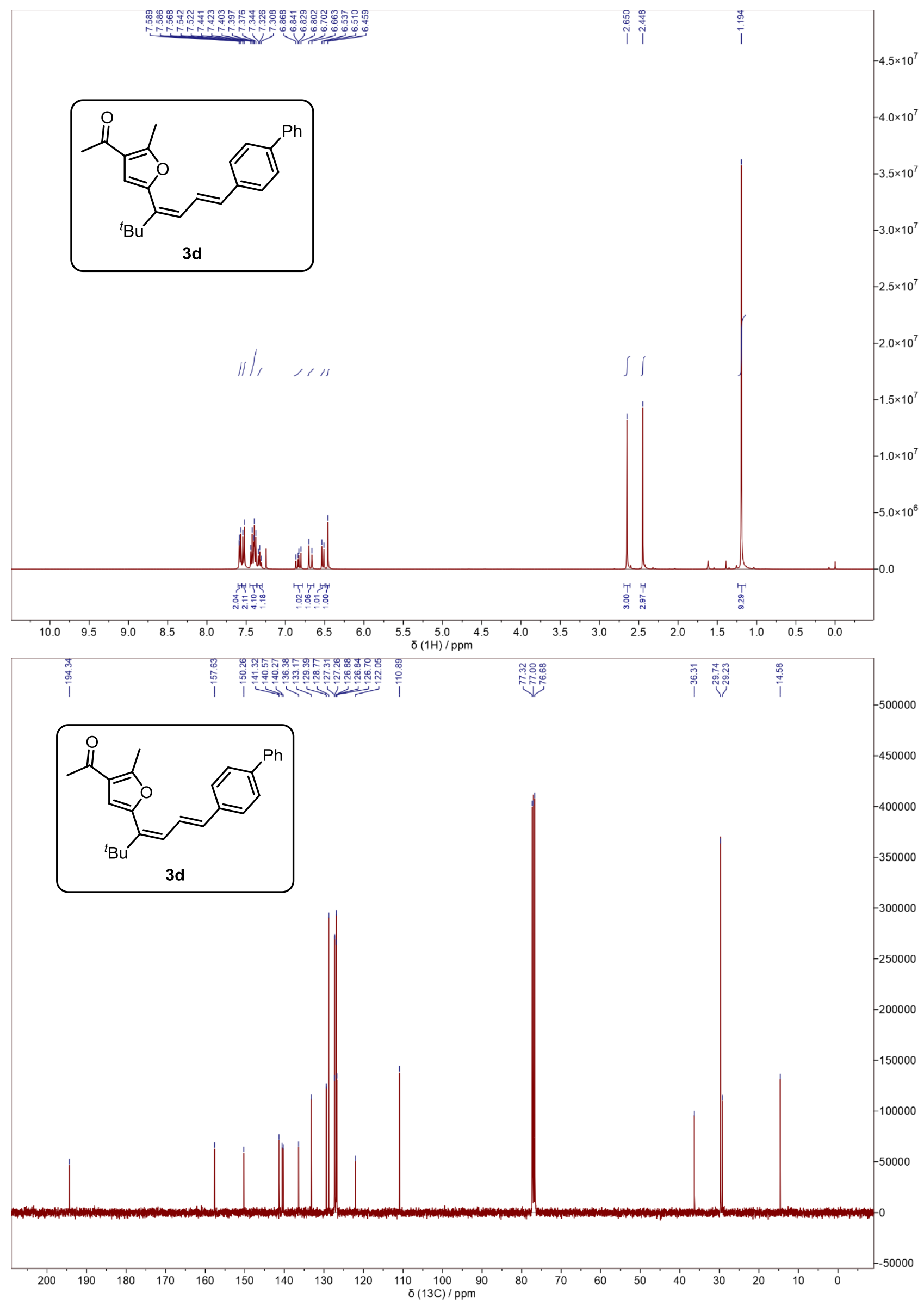




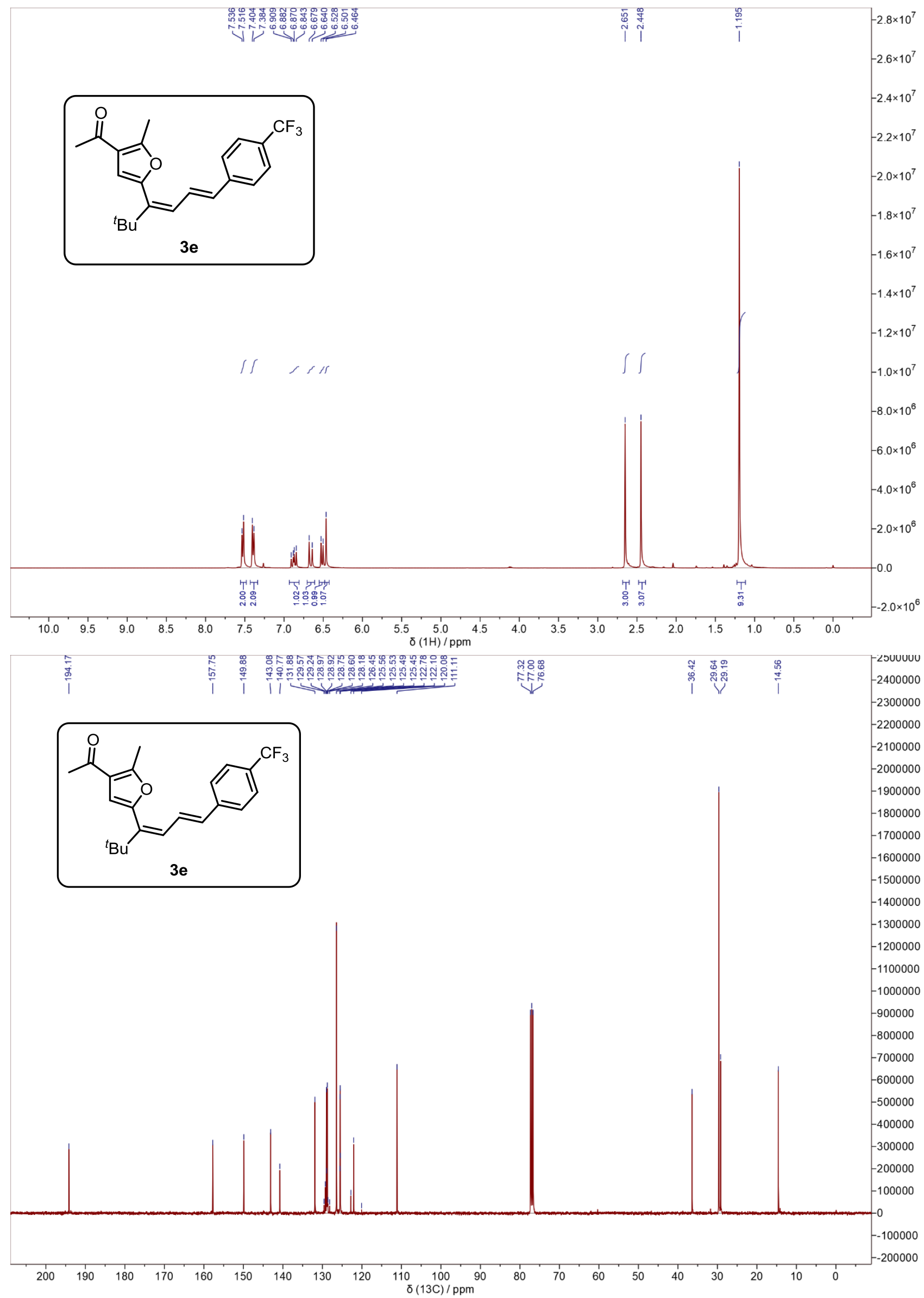

S16 


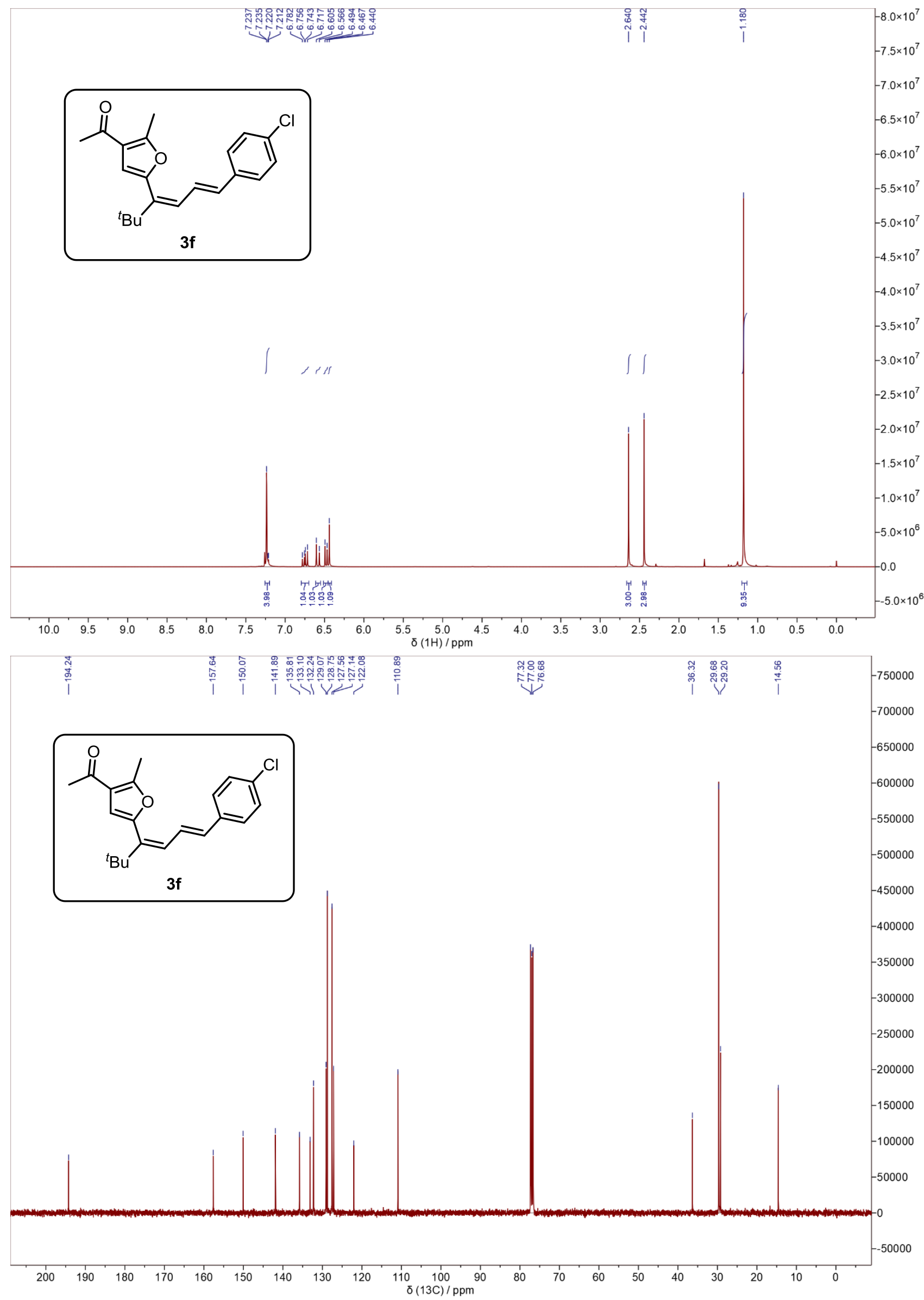




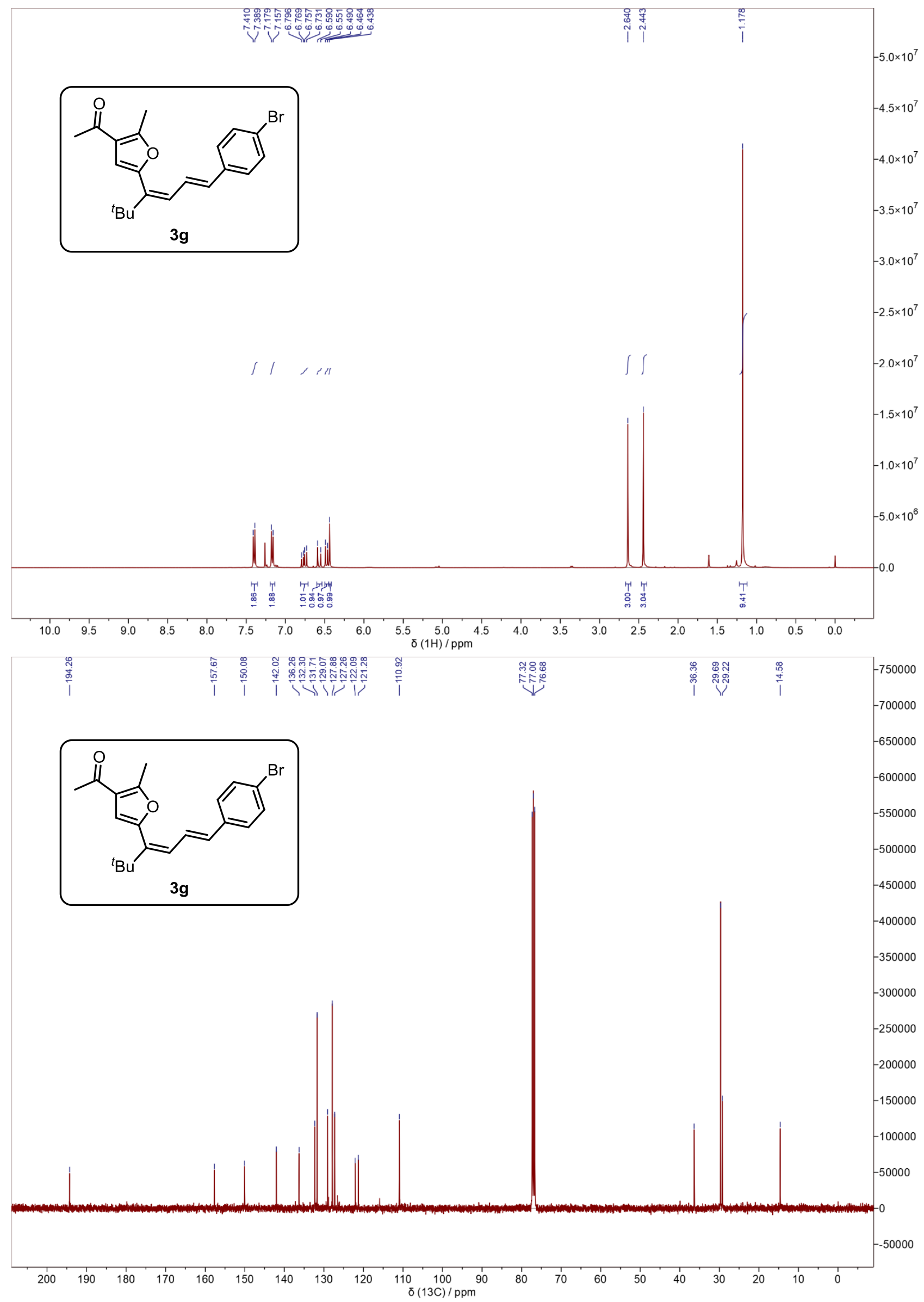




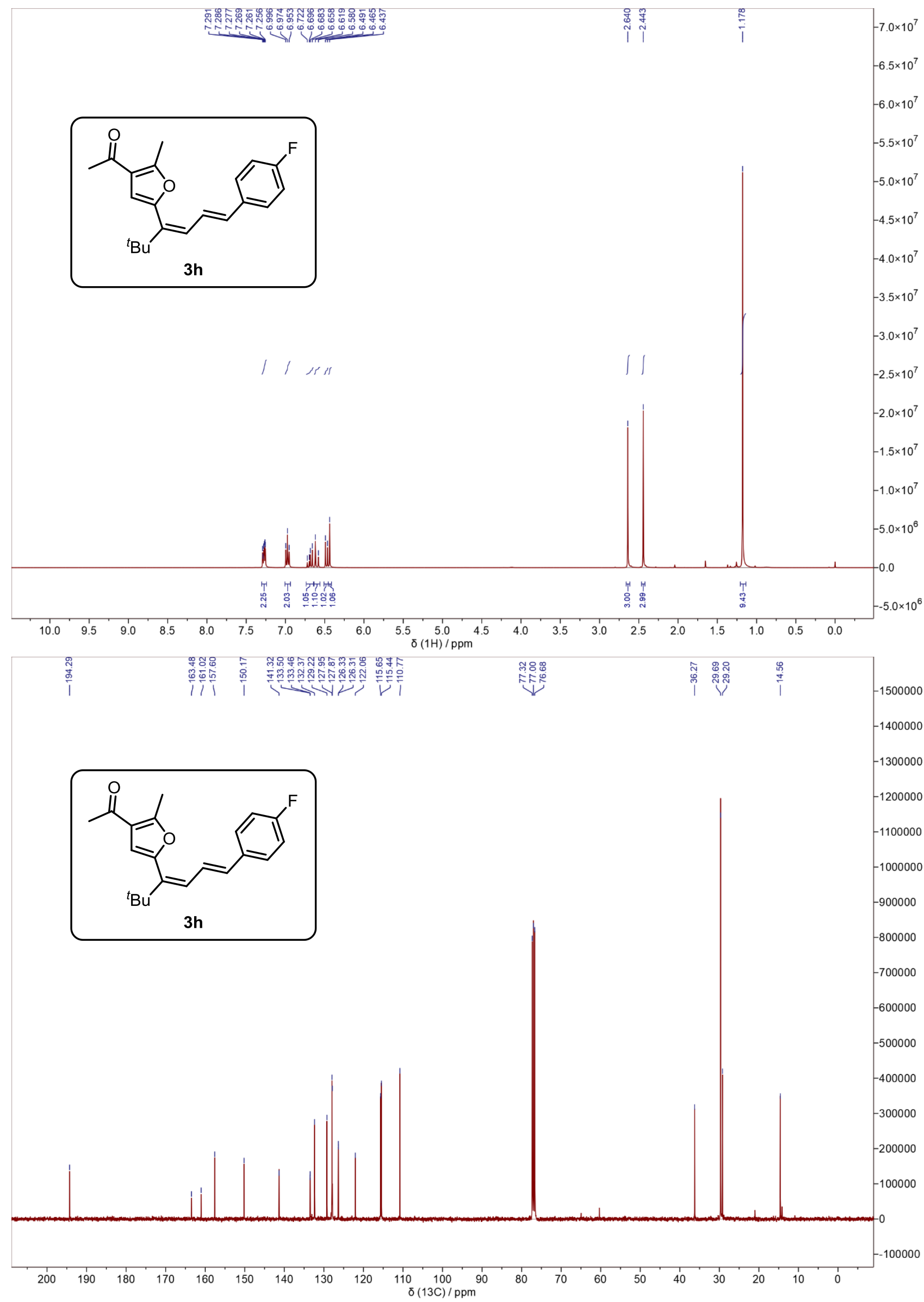




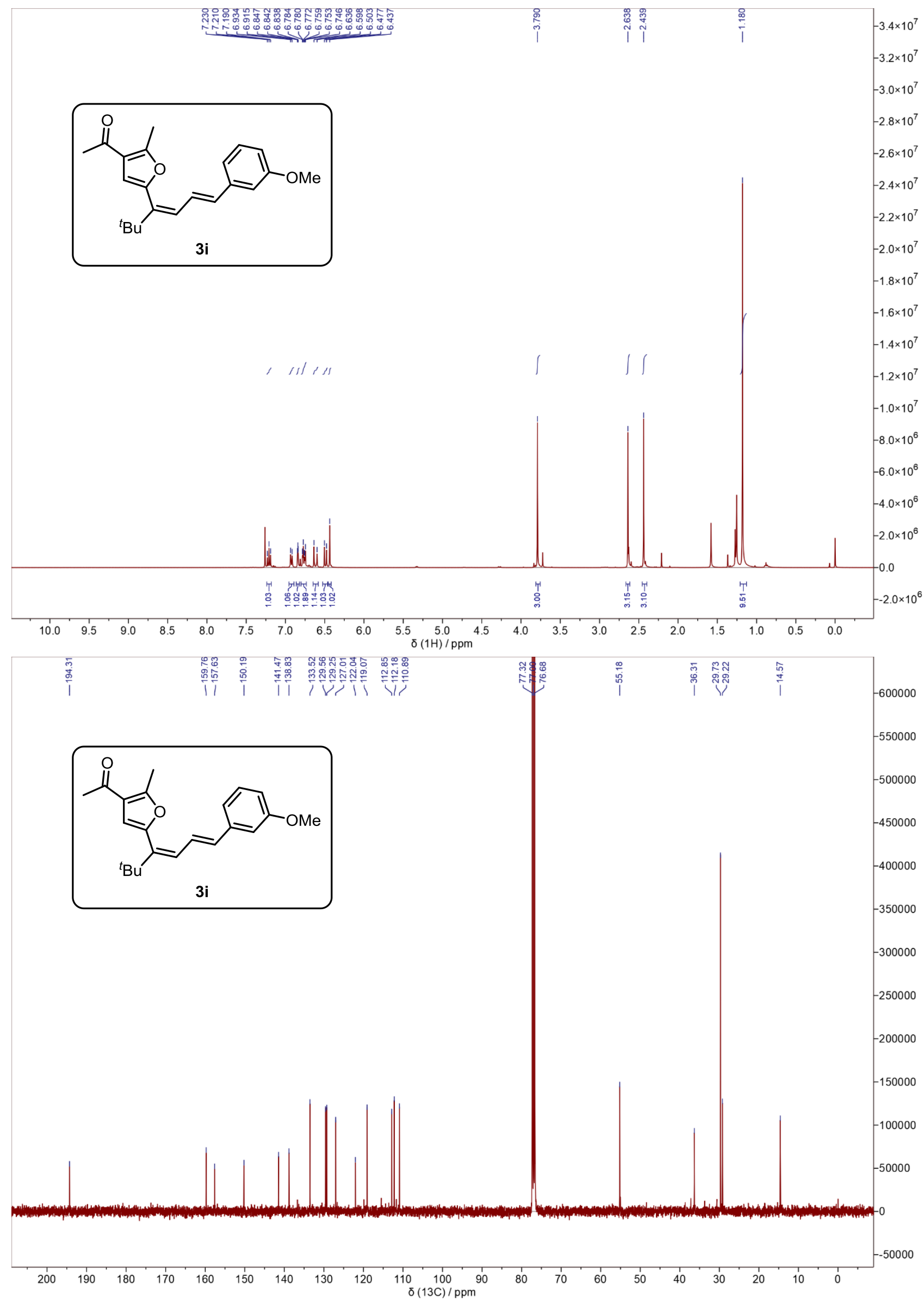




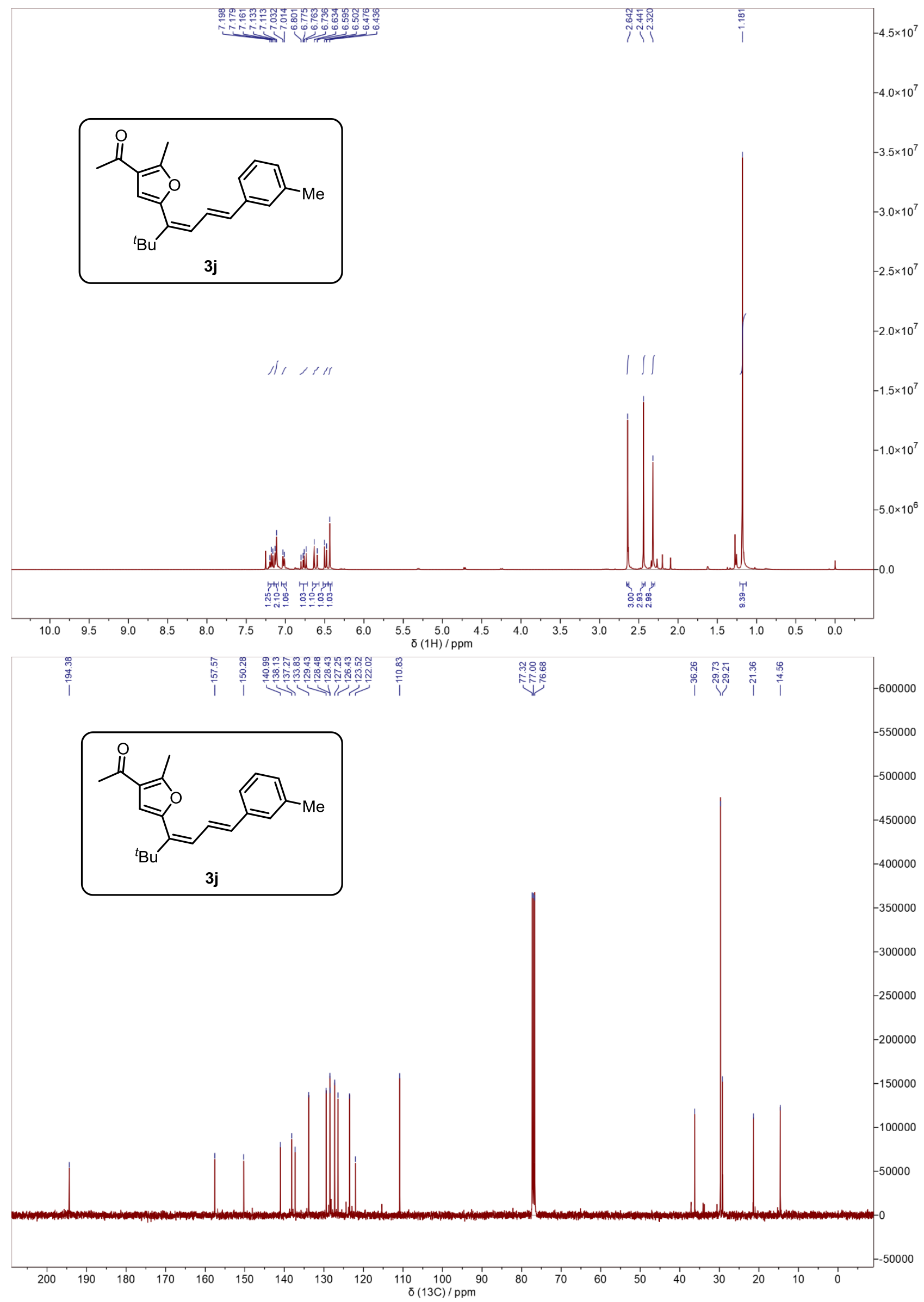




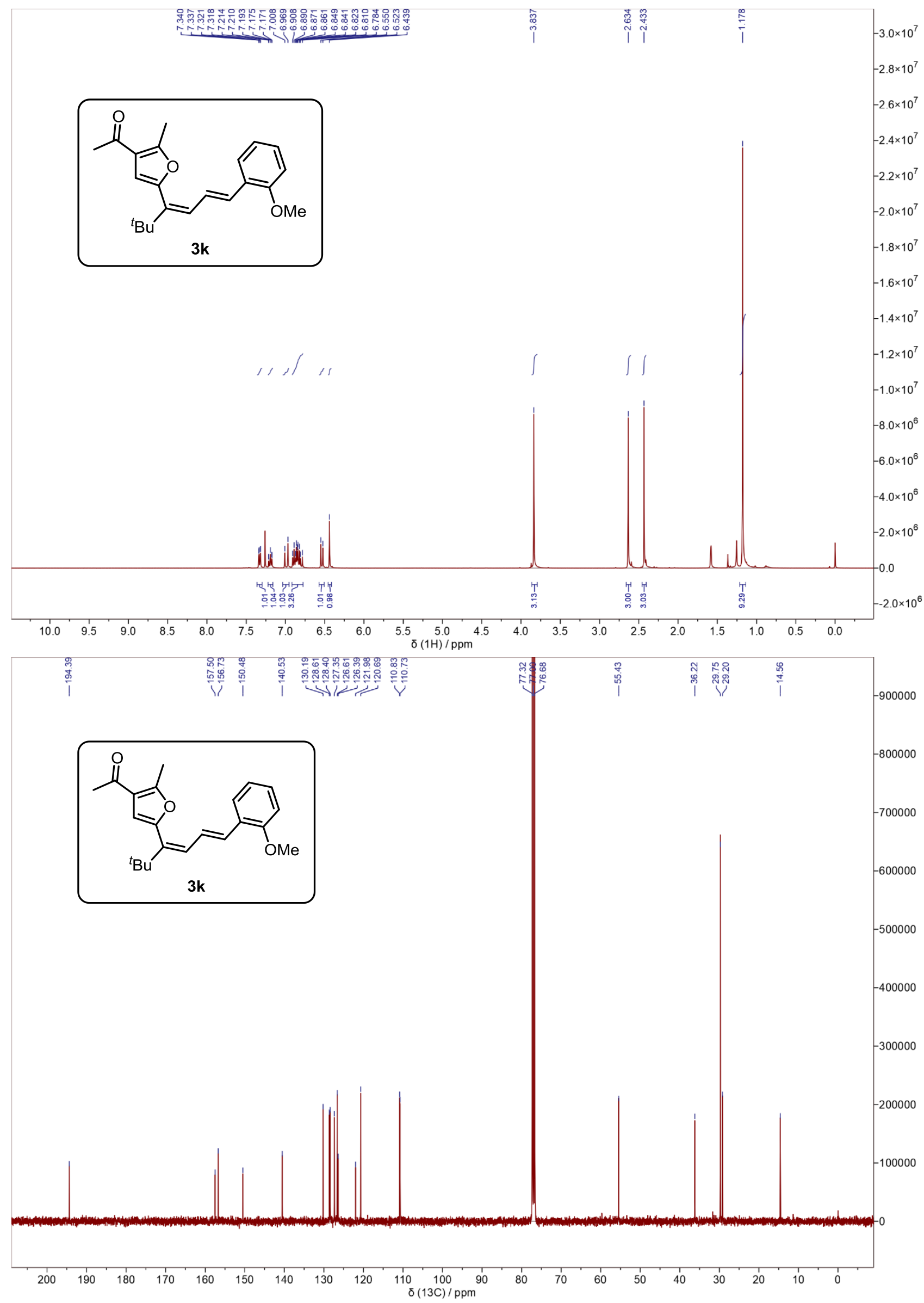




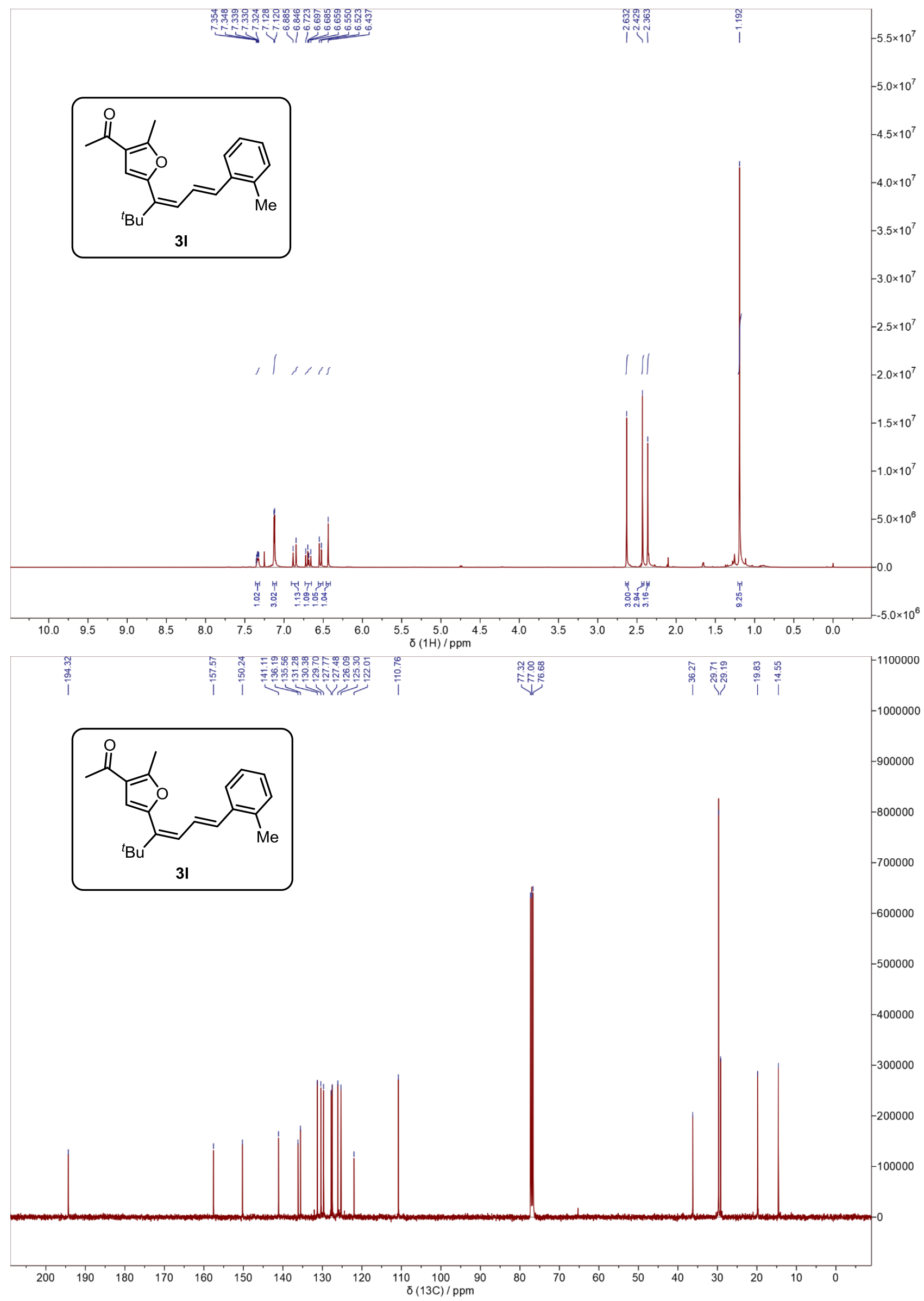




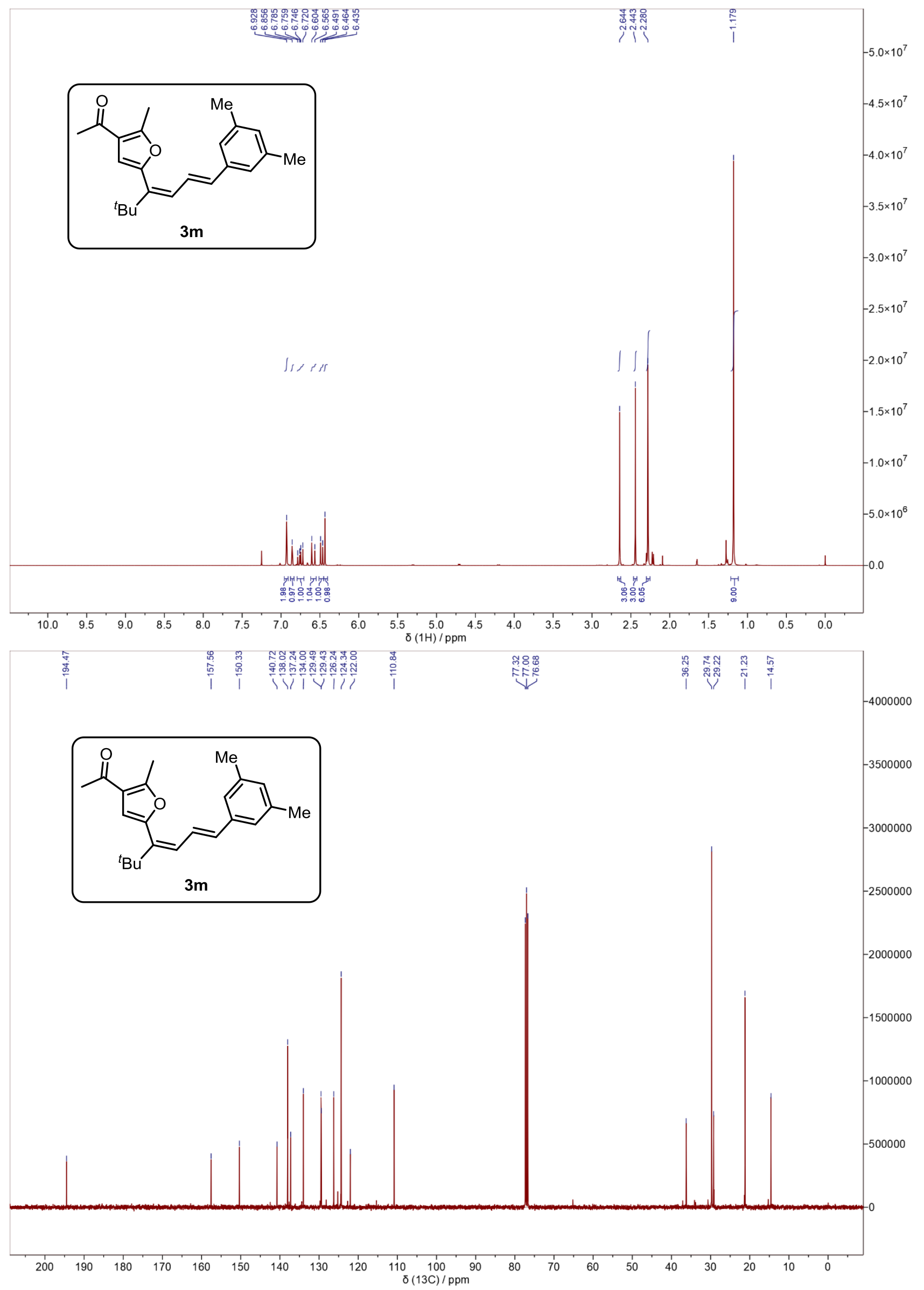

S24 


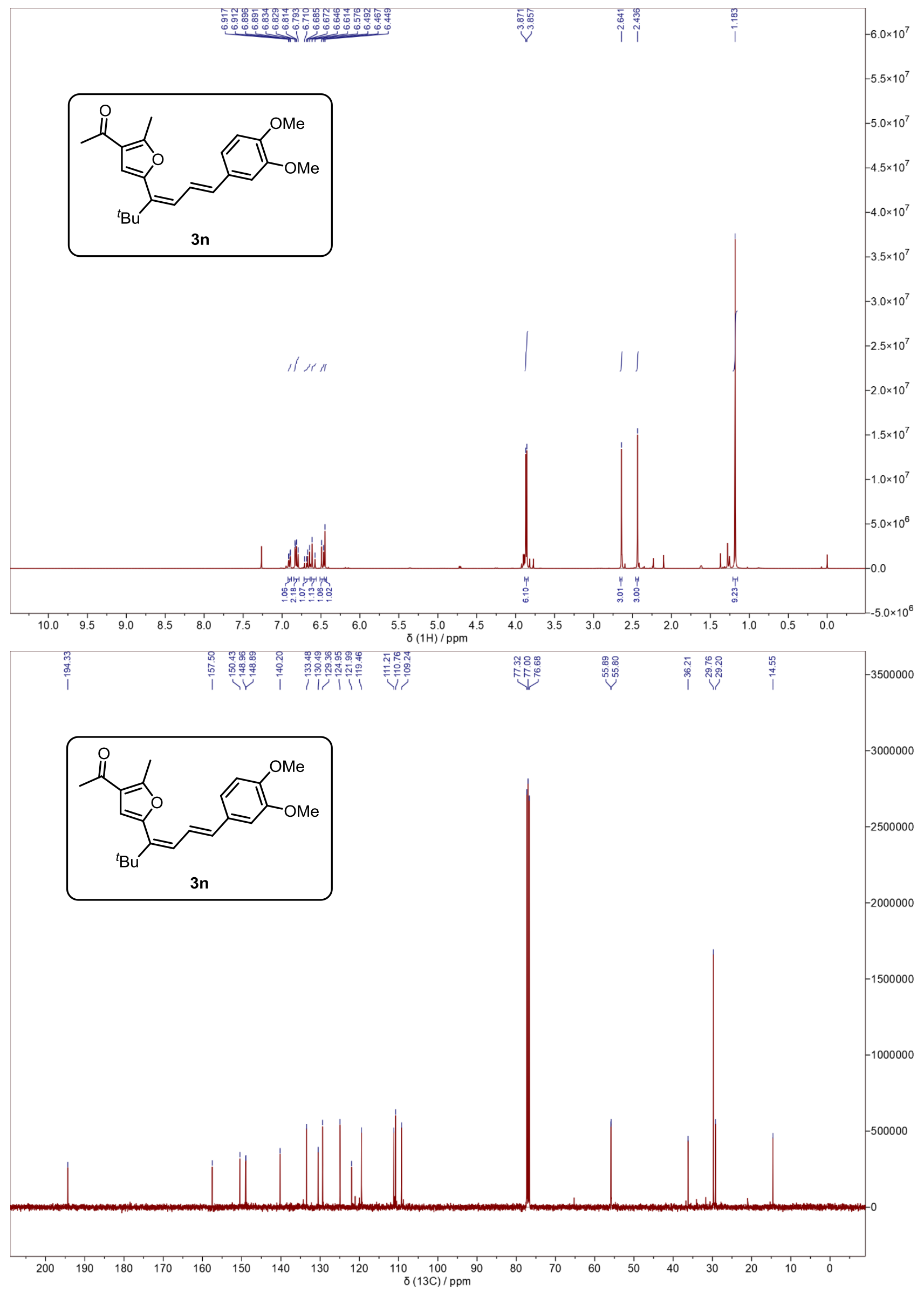

S25 


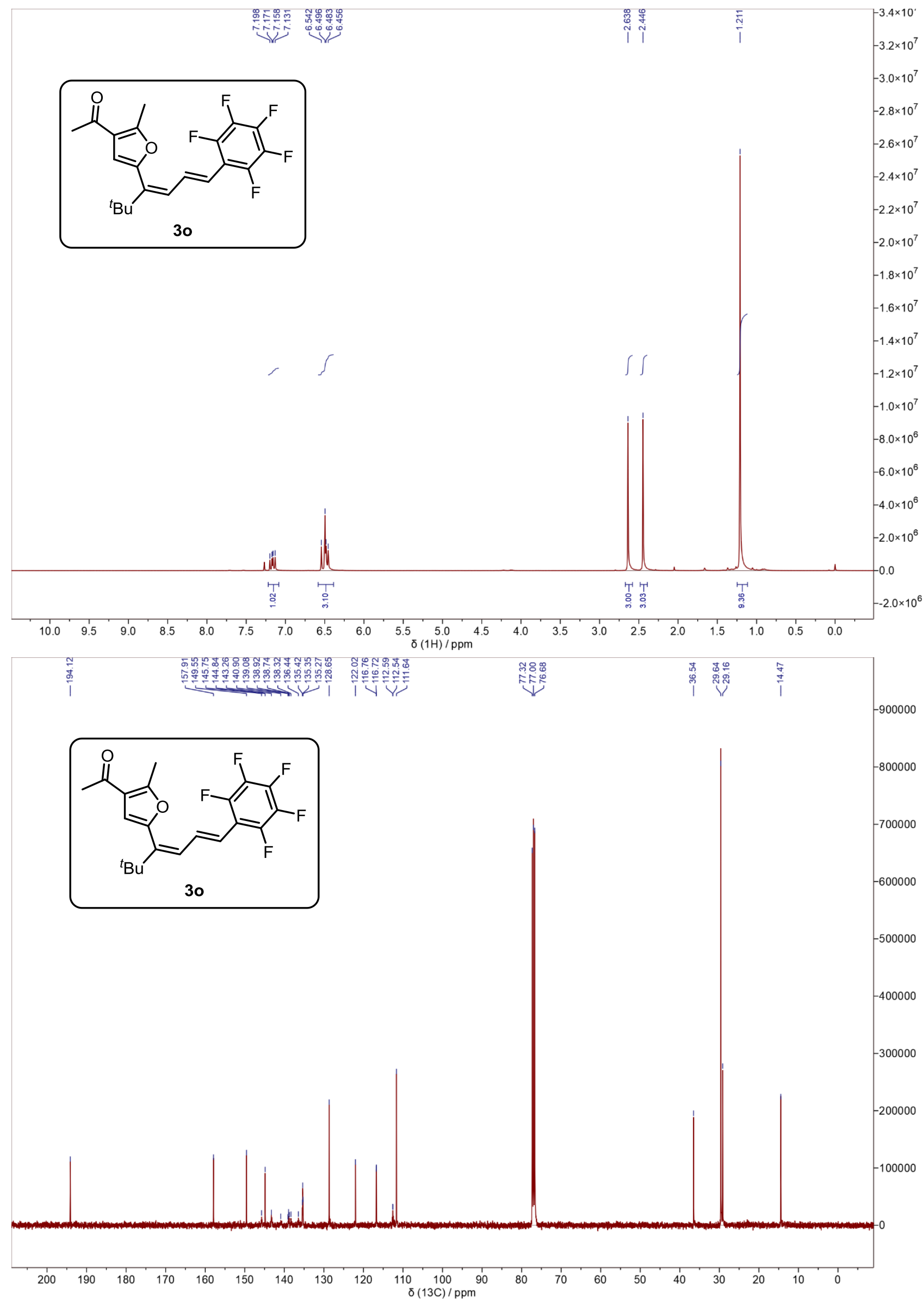

S26 


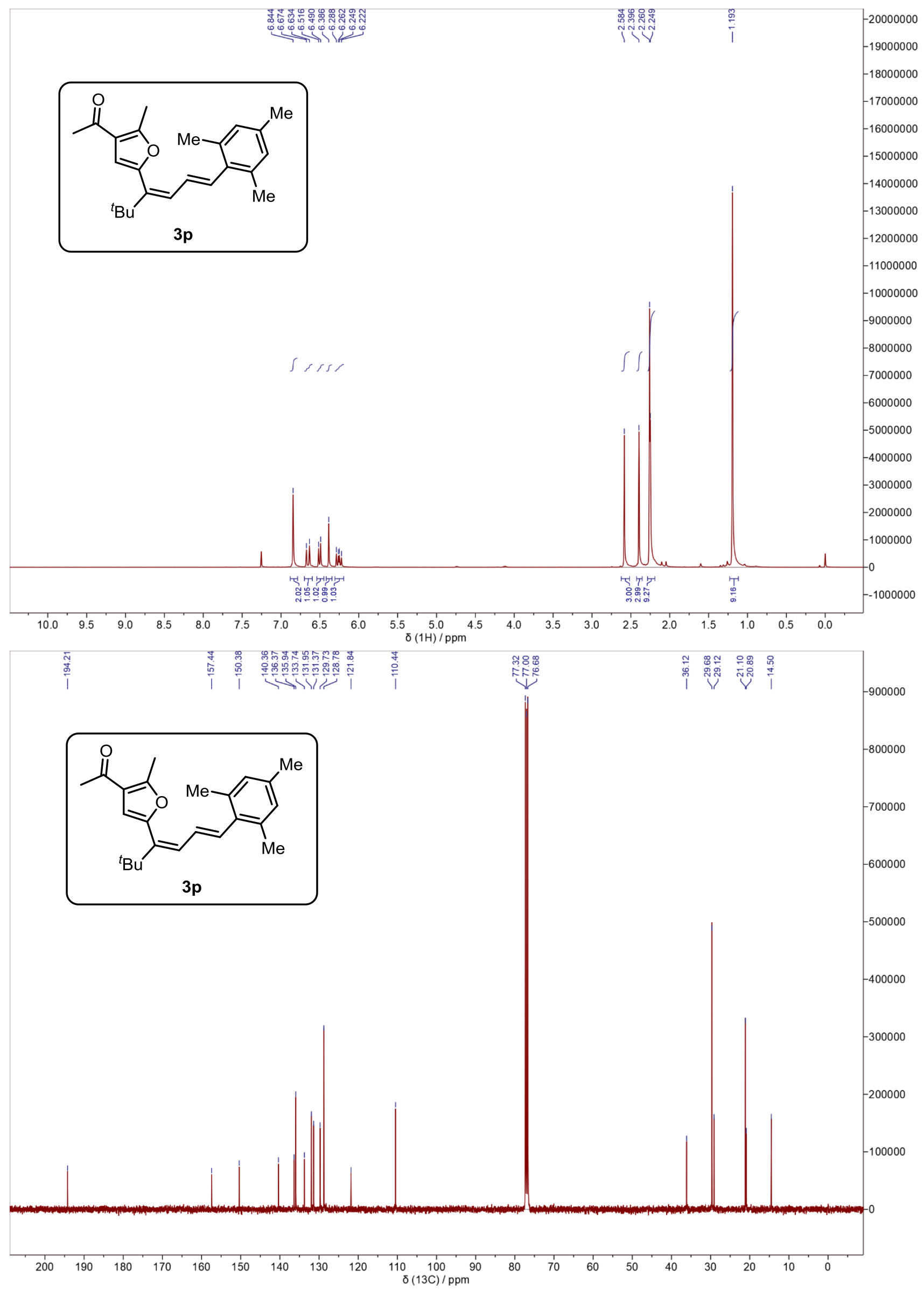




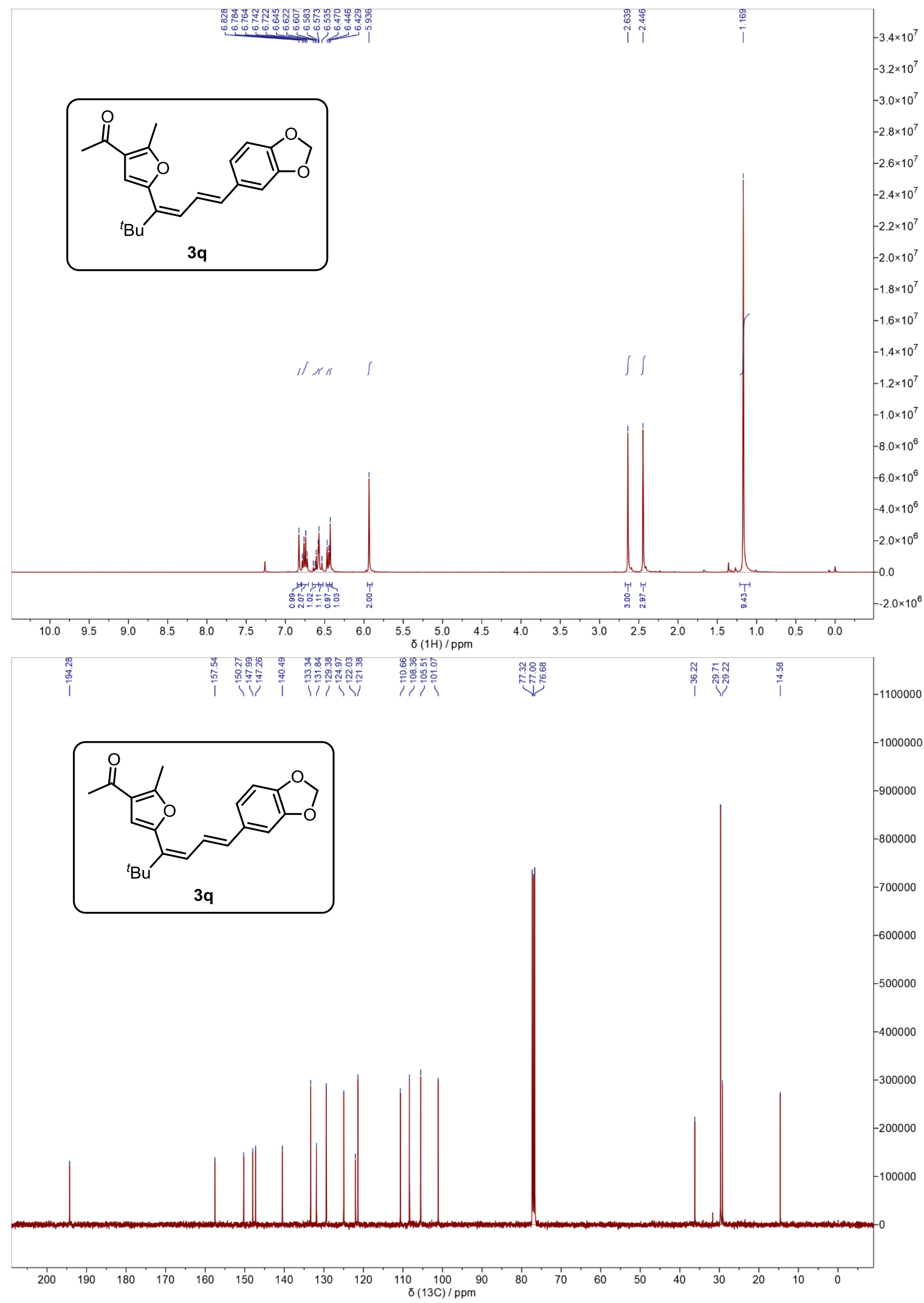




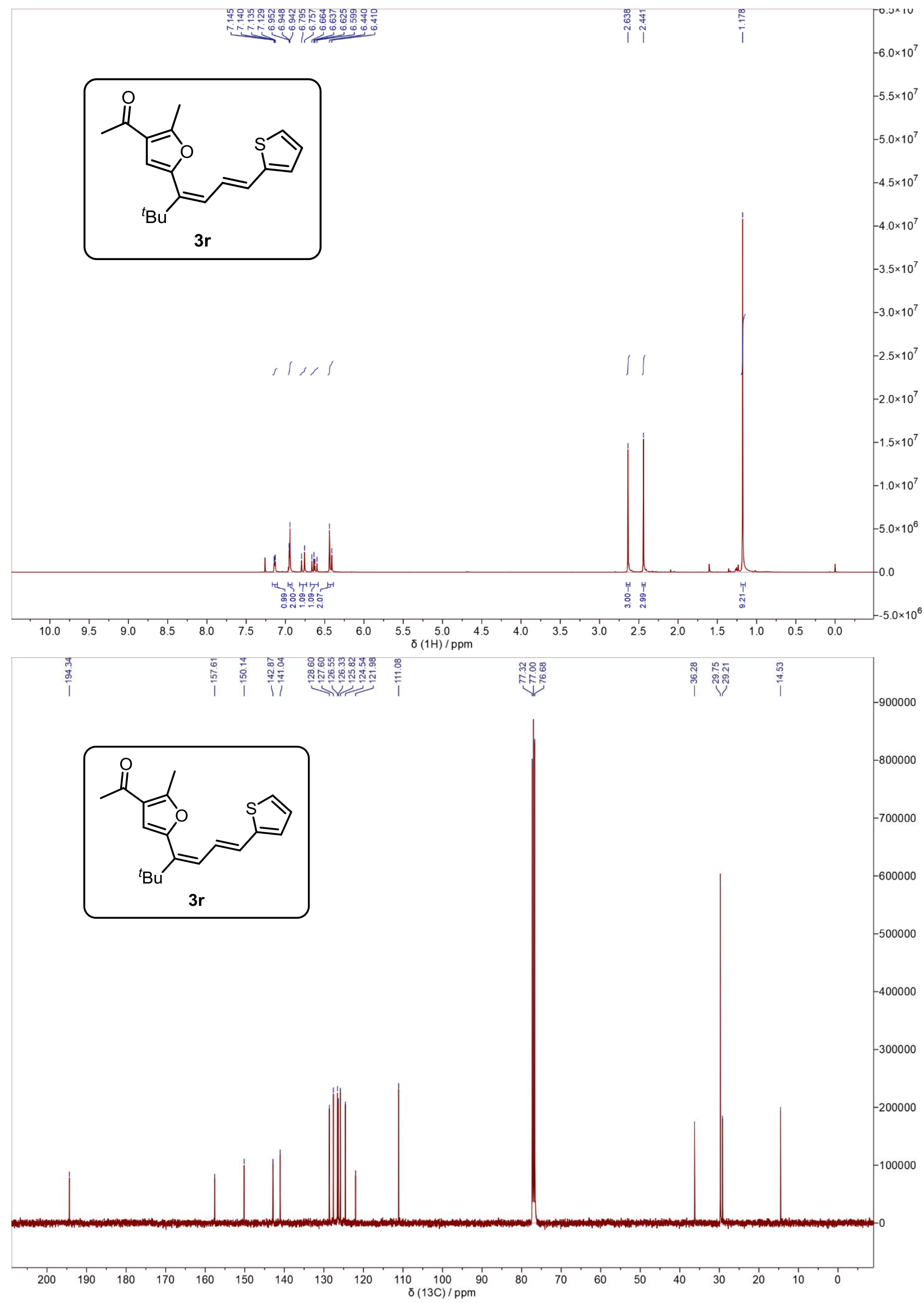




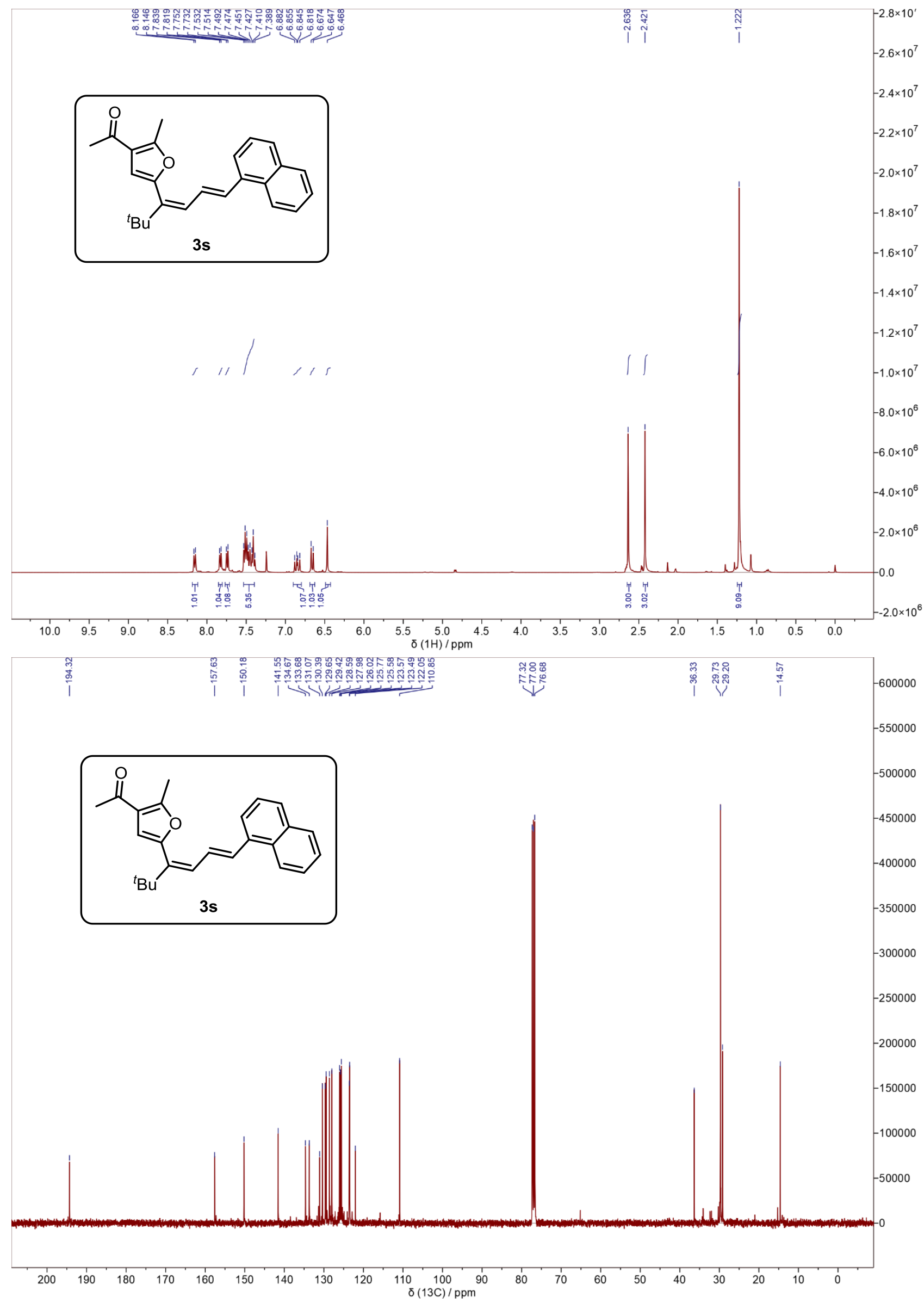




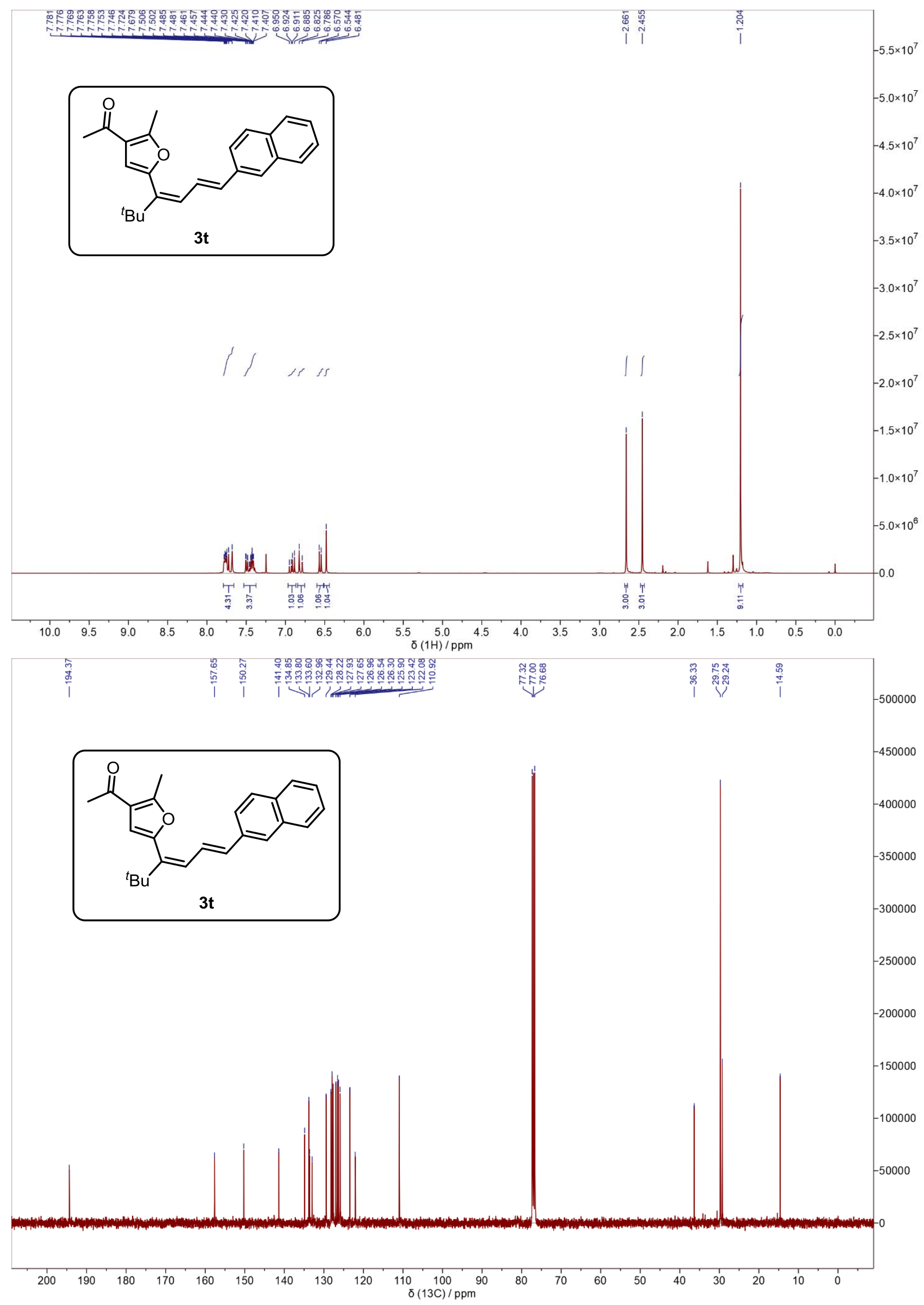




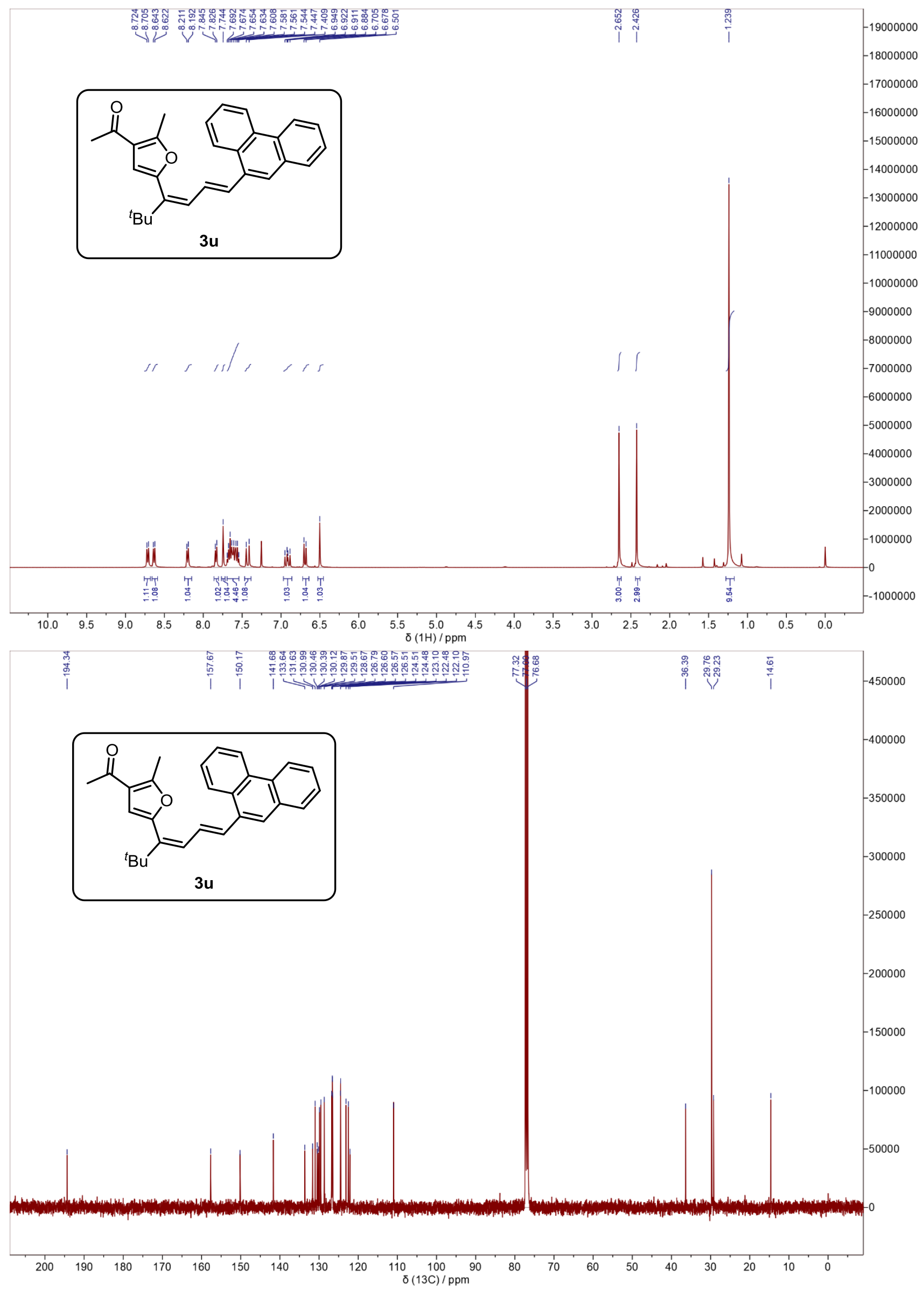




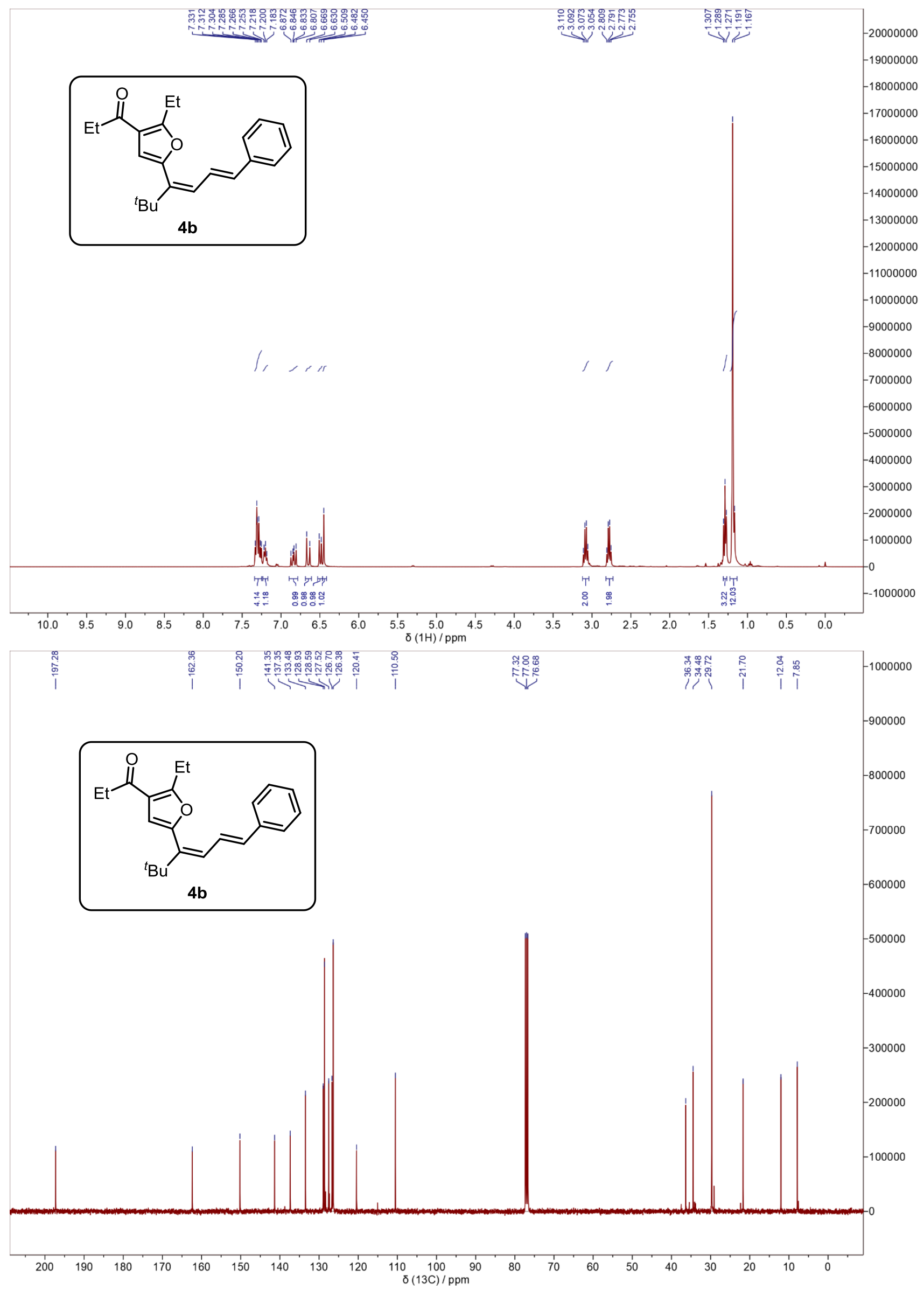




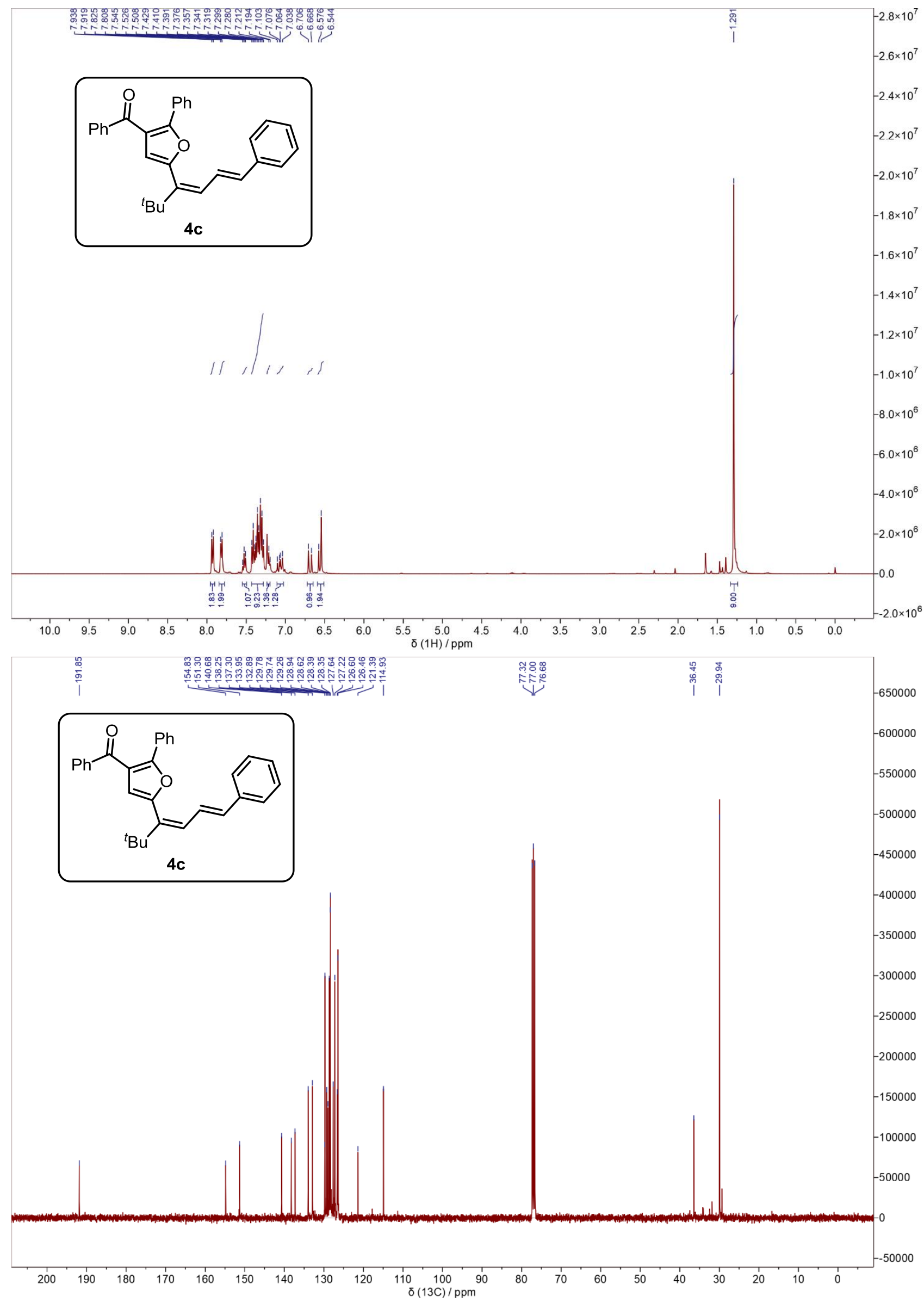




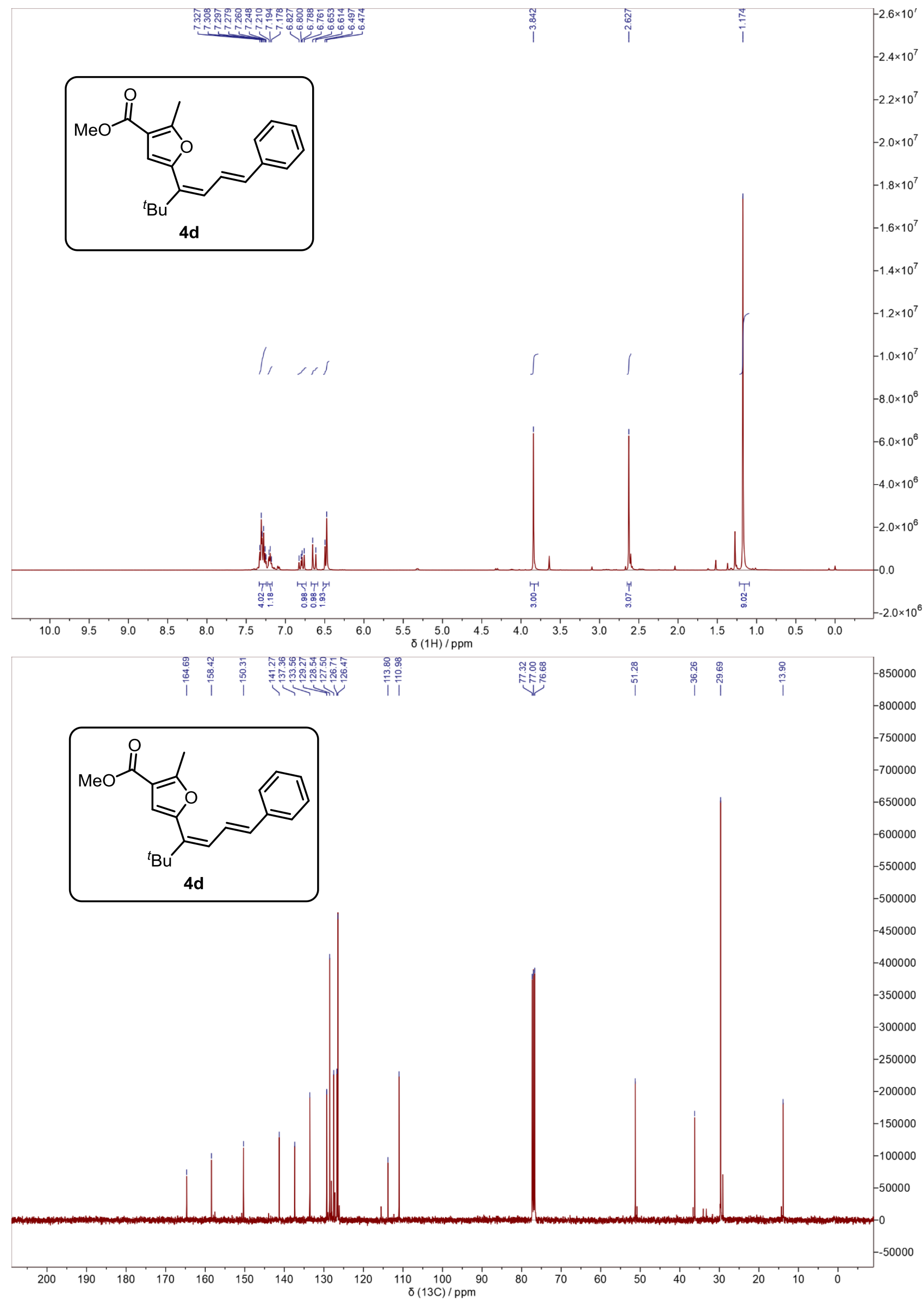




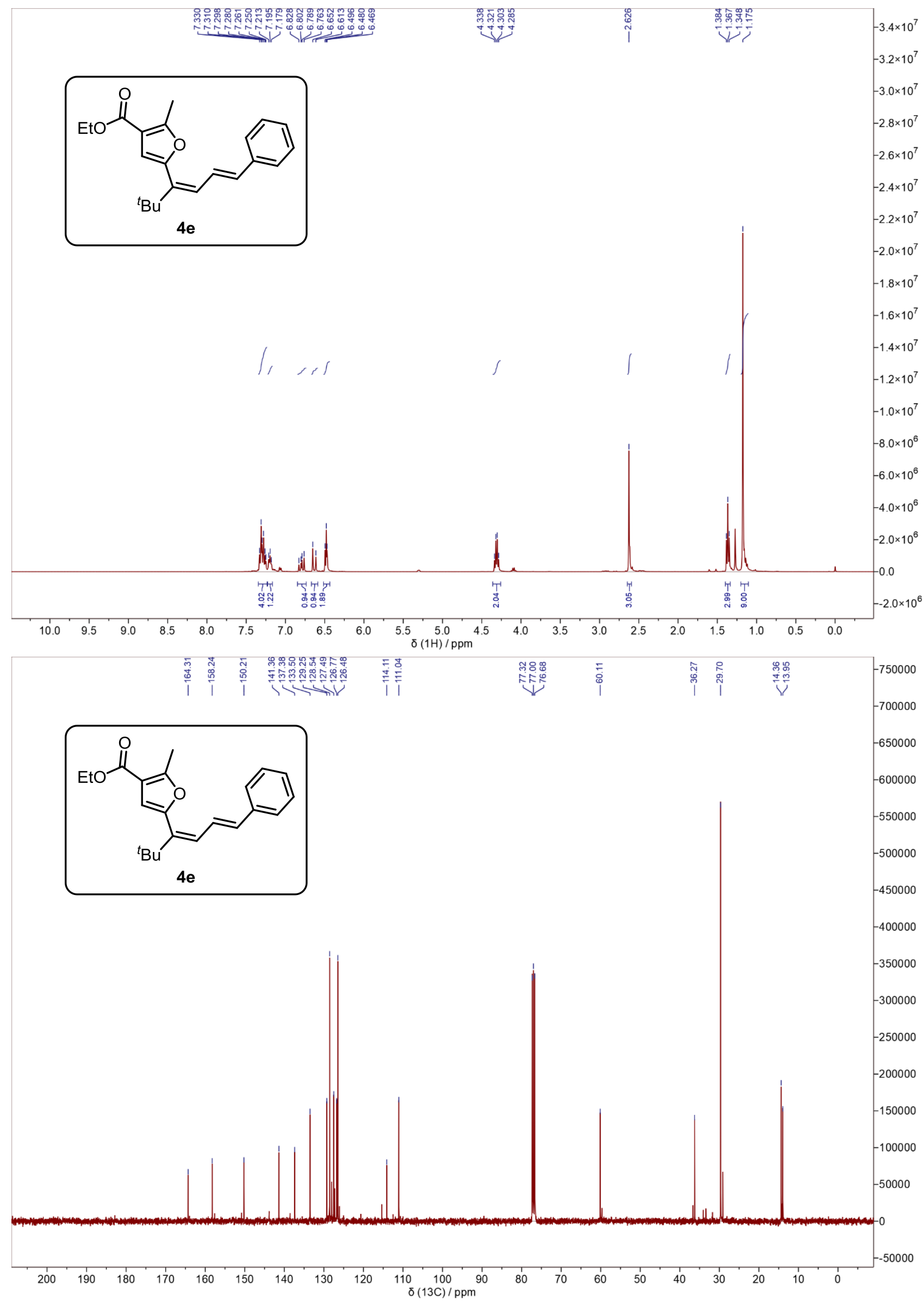




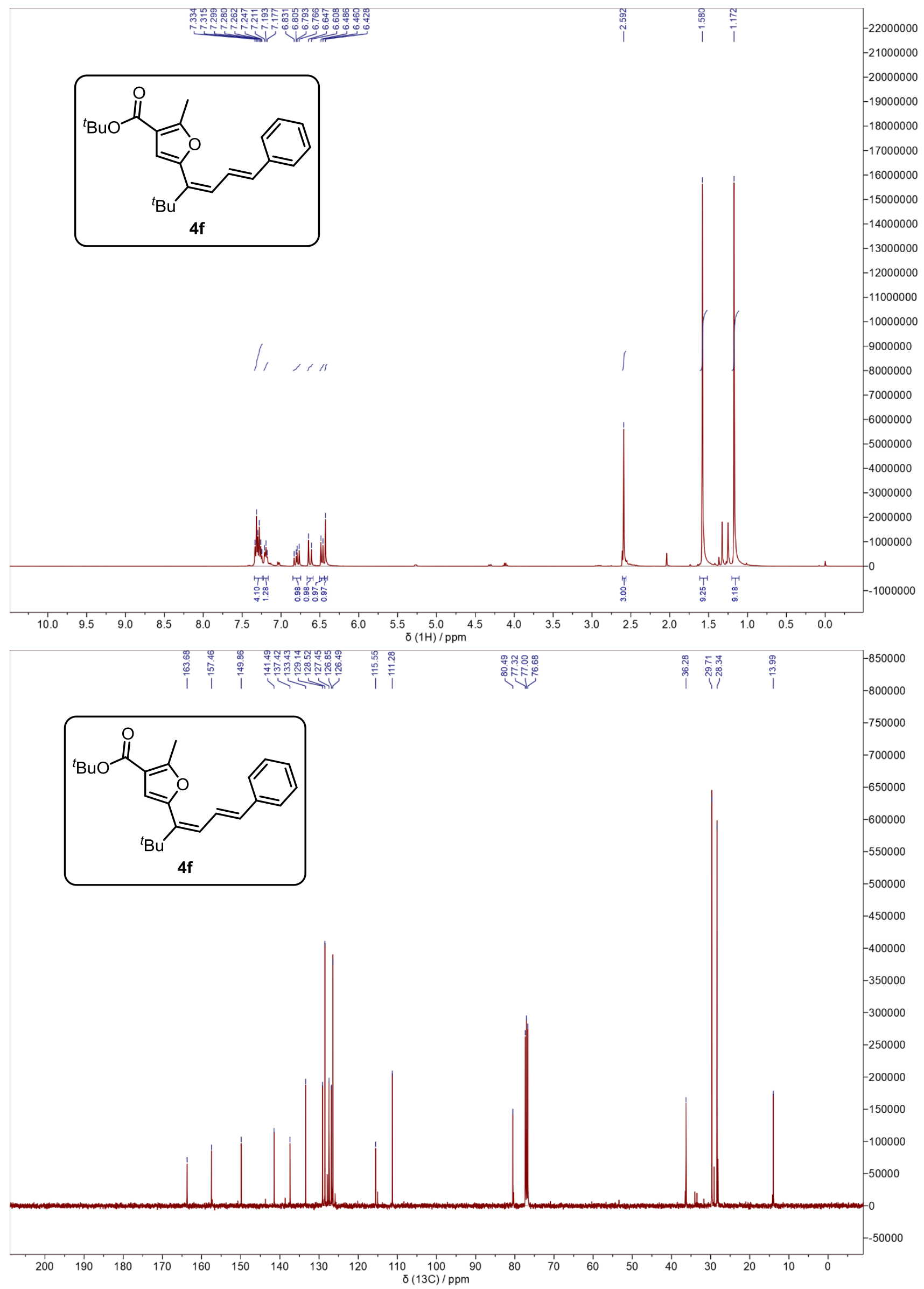




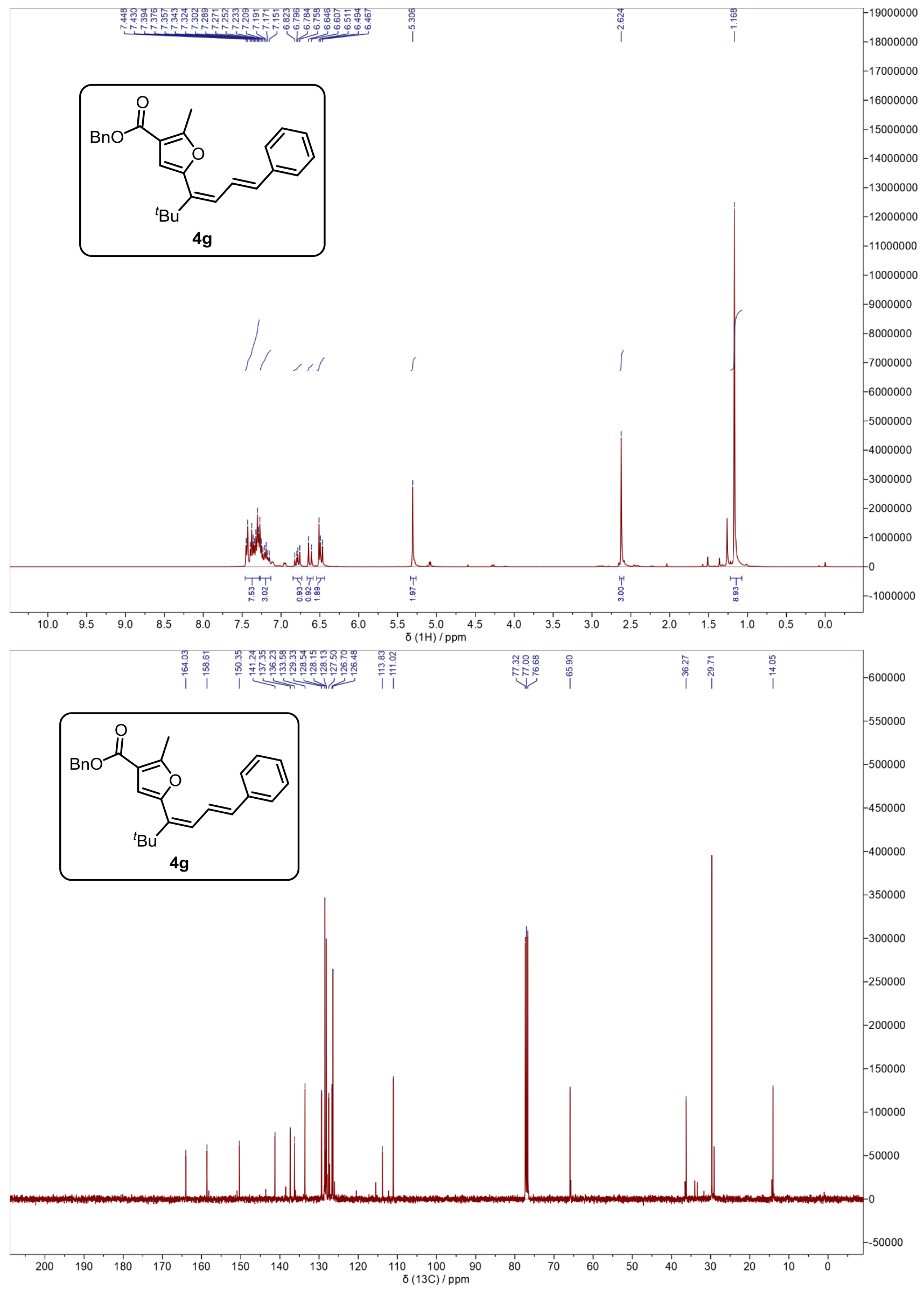




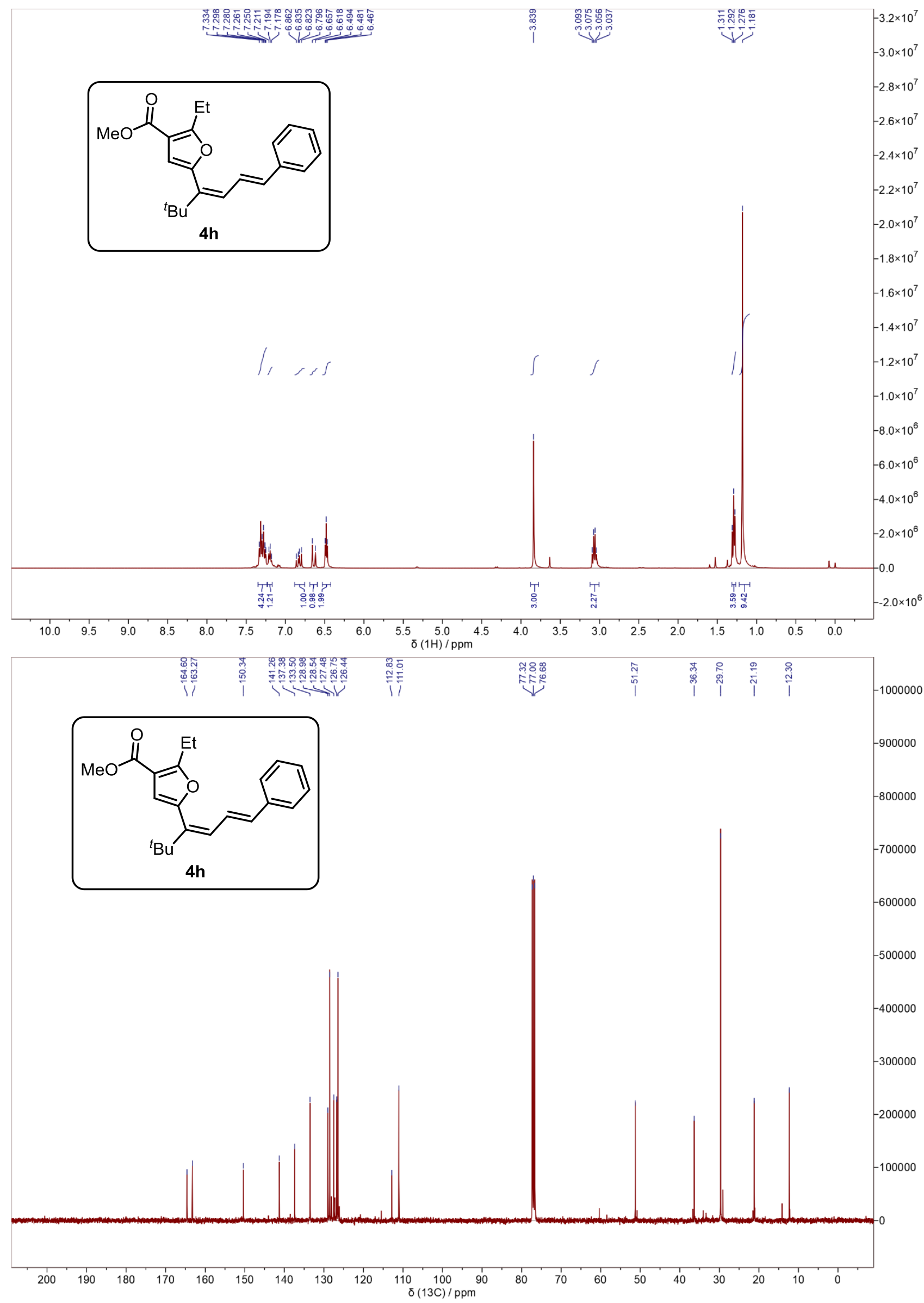




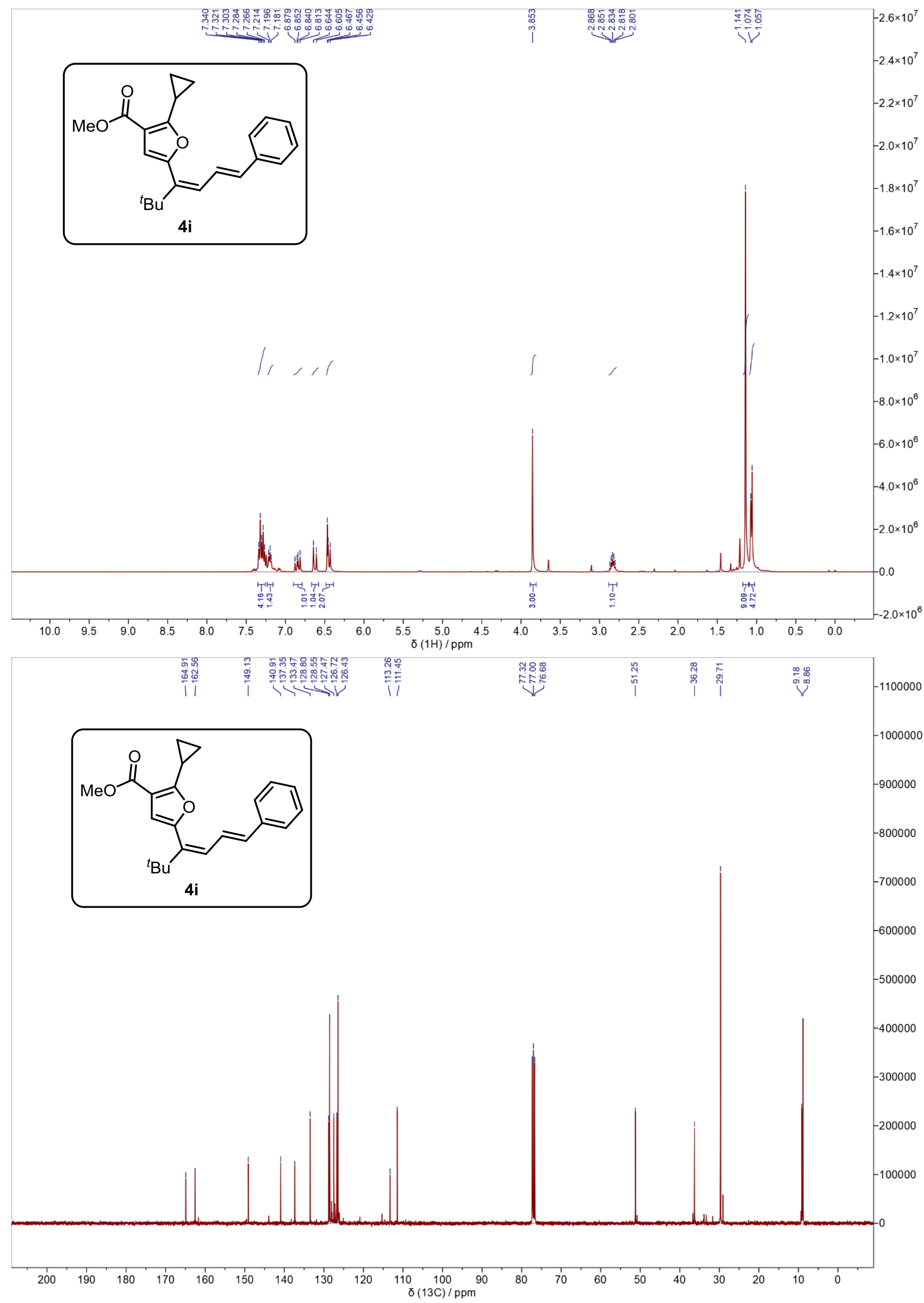




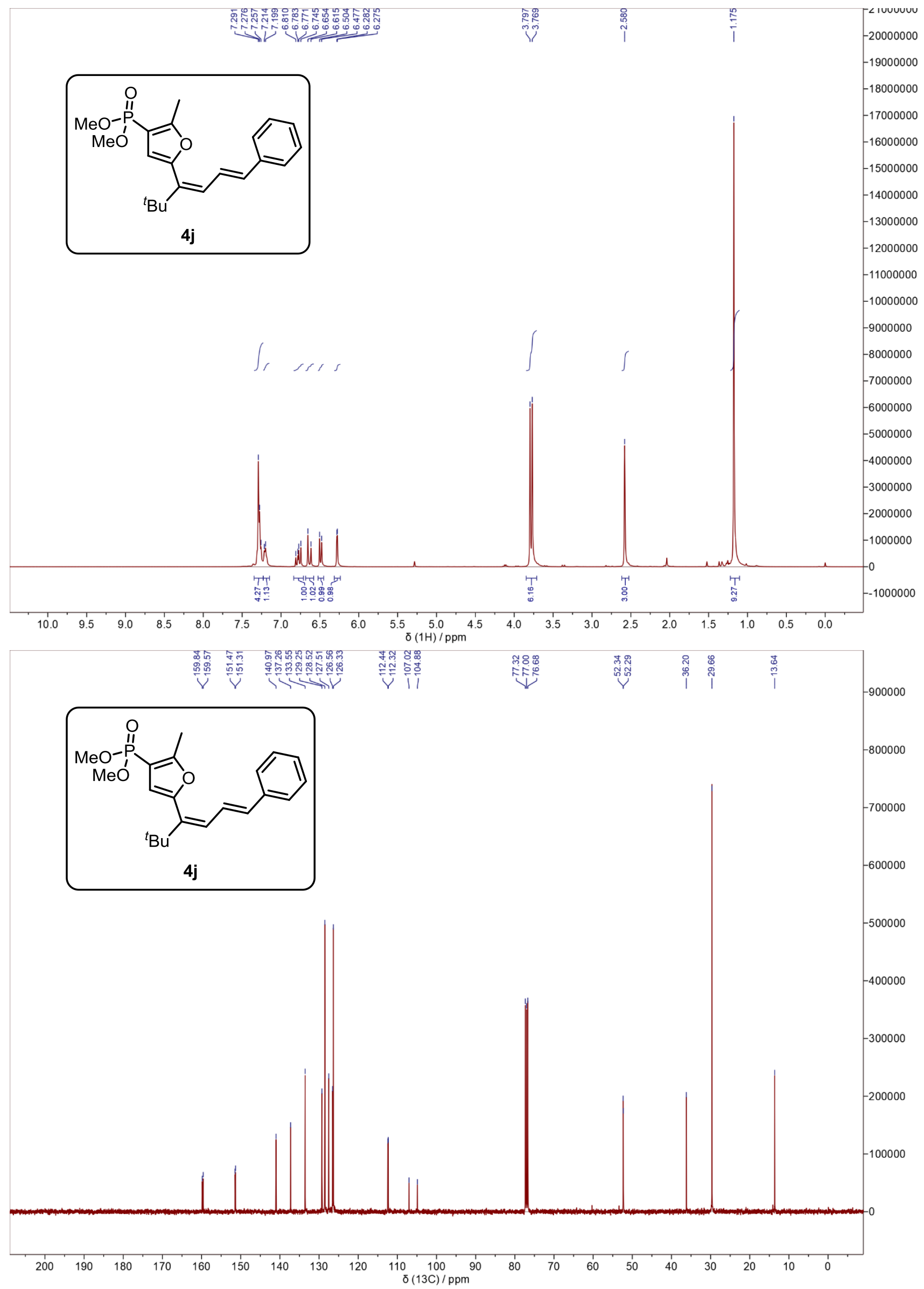




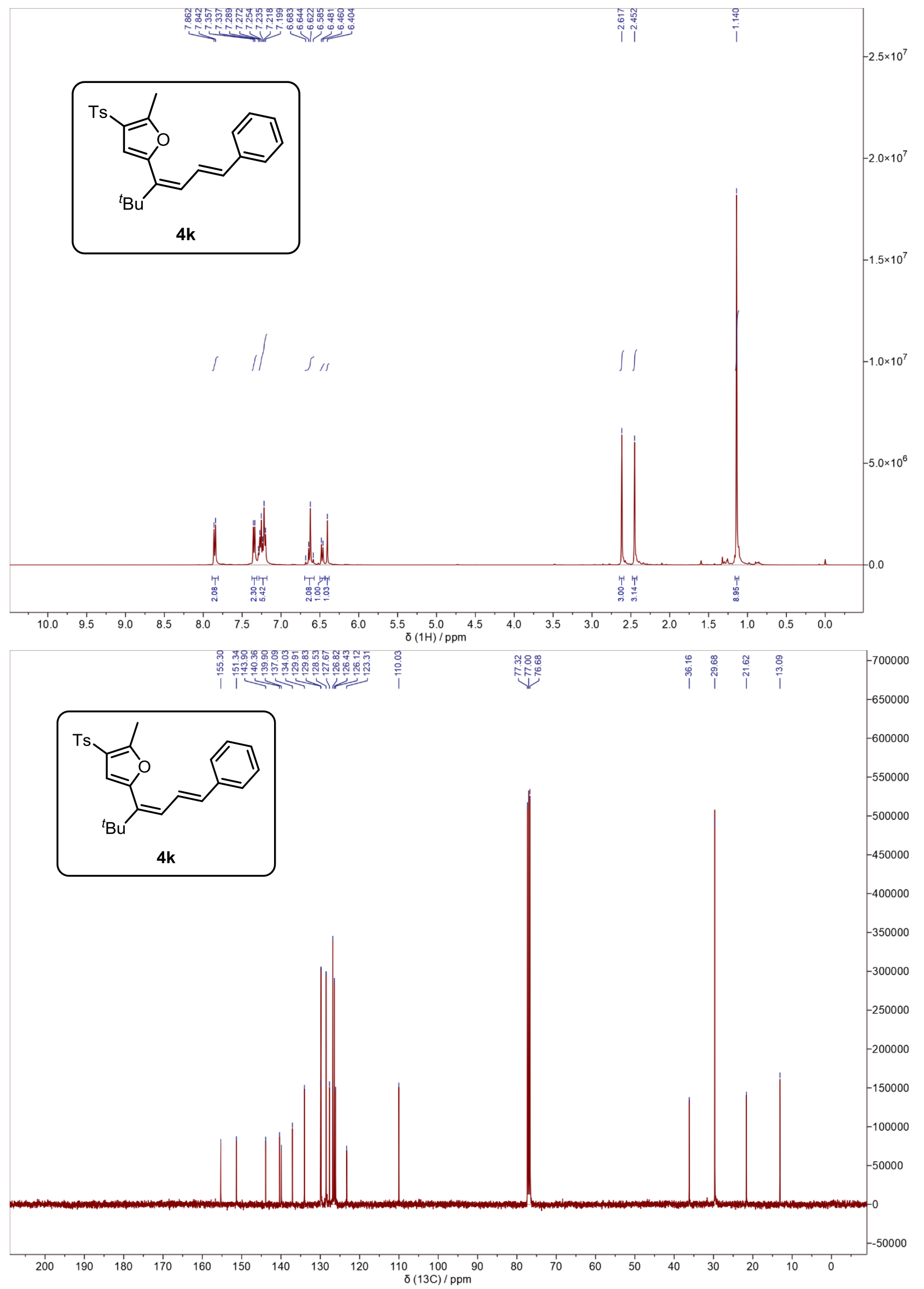

